\title{
COMBINATORIAL STRUCTURE OF TYPE DEPENDENCY
}

\author{
RICHARD GARNER
}

\begin{abstract}
We give an account of the basic combinatorial structure underlying the notion of type dependency. We do so by considering the category of all dependent sequent calculi, and exhibiting it as the category of algebras for a monad on a presheaf category. The objects of the presheaf category encode the basic judgements of a dependent sequent calculus, while the action of the monad encodes the deduction rules; so by giving an explicit description of the monad, we obtain an explicit account of the combinatorics of type dependency. We find that this combinatorics is controlled by a particular kind of decorated ordered tree, familiar from computer science and from innocent game semantics. Furthermore, we find that the monad at issue is of a particularly well-behaved kind: it is local right adjoint in the sense of Street-Weber. In future work, we will use this fact to describe nerves for dependent type theories, and to study the coherence problem for dependent type theory using the tools of two-dimensional monad theory.
\end{abstract}

\section{INTRODUCTION}

There has been much recent interest in Martin-Löf's type theory, spurred on by the discovery of remarkable links to algebraic topology and the theory of $(\infty, 1)$-categories. Homotopy type theory [23] extends Martin-Löf type theory with Voevoedsky's univalence axiom and a new collection of type-formers, the higher inductive types; the resultant system is capable of deriving key results of homotopy theory - such as calculations of homotopy groups of spheres - in a synthetic, axiomatic manner. Models of the axioms include not only classical homotopy theory, but also "non-standard homotopy theories" described by $(\infty, 1)$ toposes (homotopical analogues of categories of sheaves); in fact, it is believed that we can view homotopy type theory as providing an internal language for $(\infty, 1)$-toposes, just as first-order geometric logic does for Grothendieck toposes.

The suitability of Martin-Löf type theory as a language for abstract homotopy theory is due to the presence of identity types which can be validly interpreted by the homotopy relation. The existence of identity types relies in turn on the possibility of type dependency: type families indexed by elements of other types. While the intuitive meaning of type dependency is clear, its syntactic expression is rather involved; a desire to understand its mathematical essence

Date: October 21, 2018.

2000 Mathematics Subject Classification. Primary: 18D05, 18C15.

This work was supported by the Australian Research Council's Discovery Projects scheme, grant number DP110102360. 
has led various authors $[5,8,13,14,10,7,20]$ to describe notions of categorical model for dependent type theory which abstract away from the complexities of the syntax.

One aspect that remains implicit in both the syntactic and the categorical accounts is the combinatorial structure of type dependency: the structure imposed on the judgements of a dependent sequent calculus by the basic rules of weakening, projection and substitution. On the syntactic side, this combinatorics is hidden in the recursive clauses which generate the calculus; while on the categorical side, the essential role it plays in constructing models from the syntax is no longer visible in the finished product. In short, the syntactic approach fails to detect this structure by being insufficiently abstract, while the categorical approach fails to see it by being too abstract.

The objective of this paper is to elucidate the combinatorics of type dependency by adopting a viewpoint which is intermediate between the concrete syntactic one and the fully abstract categorical one. We will model dependent sequent calculi as algebras for a monad on a presheaf category (we assume the reader is familiar with the basic concepts of category theory as set out in [19]). Objects of the presheaf category will encode the basic judgement-forms of a sequent calculus; the algebraic structure imposed on them by the monad will encode the deduction rules. Now the combinatorial structure we wish to describe inheres in the action of the monad, and so by giving an explicit description of this action, we obtain an explicit account of the structure. More precisely, the underlying endofunctor of the monad describes the derivable judgements of a freely-generated sequent calculus; while the monad multiplication encodes the process of proof-tree normalisation by which such derivations are combined.

For the dependent sequent calculi to be studied in this paper, we will not consider rules for type-formers such as $\Pi$-types, $\Sigma$-types and identity types, but rather concentrate on the core structural rules of weakening, projection and substitution. The combinatorics arising from just these rules is particularly elegant; we will see that in a freely-generated theory of this kind, the shape of derivable judgements is controlled by suitably decorated heaps. A heap is a finite tree with a total order on its nodes refining the tree order. This structure is common throughout logic and computer science, and the manner in which it appears here is highly reminiscent of its role in the study of (logical) game semantics and innocent strategies [9]. We hope to explore this link further in future work.

Beyond elucidating a structure which we believe to be interesting in its own right, the approach taken in this paper will also enable the analysis of dependent type theory using the tools of combinatorial category theory. This is a particular strand of category theory, growing out of Joyal's work [15], which has found recent applications $[18,25,16,26]$ in taming some of the complexities of higherdimensional category theory. A central theme in combinatorial category theory is the study of monads possessing abstract categorical properties that allow them to be seen as fundamentally combinatorial in nature. It turns out that the 
monad for dependent type theories is of this kind. More precisely, it is local right adjoint or familially representable in the sense of $[4,21,17]$ : and this permits the application of a rich body of theory [24, 18, 25, 2] concerning such monads to the study of dependent type theories. It is beyond the scope of this paper to investigate these connections in detail but let us mention two applications we intend to pursue in future work; see Section 8 below for a more detailed sketch of these applications.

Firstly, we will apply the results of [25] to describe a nerve functor for dependent sequent calculi: thus, a fully faithful embedding of the category of dependent sequent calculi into a presheaf category, together with a characterisation of the objects in the image. We hope to use this nerve functor to reveal an implicit geometry of dependent sequent calculi. Secondly, we will provide a new take on the coherence problem [11] for dependent type theory. We will do so by lifting the monad for dependent type theories to a 2-monad on a presheaf 2-category, and studying its pseudoalgebras, which are abstract presentations of Curien's "syntax with substitution up to isomorphism" [6].

We now give an overview of the contents of the paper. We begin in Section 2 with a description of the syntax of the dependent sequent calculi of interest to us, which are the generalised algebraic theories of [5]; we also describe the interpretations between two such theories, so yielding the objects and morphisms of the category GAT of generalised algebraic theories.

In Section 3, we begin our combinatorial analysis of the category GAT by describing a presheaf category $\left[\mathcal{H}^{\mathrm{op}}\right.$, Set $]$ whose objects encode the basic judgements of a generalised algebraic theory. We define a forgetful functor GAT $\rightarrow$ $\left[\mathcal{H}^{\text {op }}\right.$, Set $]$, and show that this has a left adjoint and is monadic. The main goal of the paper will be to give an explicit description of the induced monad.

In fact it will be convenient - and illuminating - to split this task up. Writing $w, p$, and $s$ for the weakening, projection and substitution rules, we consider for each $D \subset\{w, p, s\}$ the category $D$-GAT of "generalised algebraic theories with structural rules from $D$ ". Again, each forgetful functor $D$-GAT $\rightarrow\left[\mathcal{H}^{\text {op }}\right.$, Set $]$ has a left adjoint and is monadic, and by studying the induced monads for various choices of $D$, we may understand the structure induced by various combinations of the three rules.

In Section 4 , we consider the structure imposed on $\left[\mathcal{H}^{\text {op }}\right.$, Set $]$ by the rule of weakening alone, without projection or substitution; thus, we characterise the monad $W$ induced by the forgetful functor $\{w\}$-GAT $\rightarrow\left[\mathcal{H}^{\text {op }}\right.$, Set $]$. Then in Section 5, we consider the structure imposed by the projection rule. As this rule in fact relies on the weakening rule for its well-formedness, we are forced to consider both together: we thus describe the monad $P$ induced by the functor $\{w, p\}$-GAT $\rightarrow\left[\mathcal{H}^{\text {op }}\right.$, Set $]$. In Section 6 , we go on to consider the structure imposed by substitution alone, which involves describing the monad $S$ induced by the forgetful functor $\{s\}$-GAT $\rightarrow\left[\mathcal{H}^{\text {op }}\right.$, Set $]$; and then in Section 7 , we combine together the monads for weakening and projection and for substitution into a 
compound monad $T=P S$ for generalised algebraic theories. The extra datum required to do so is a distributive law in the sense of [1] between the two monads $P$ and $S$; this distributive law describes the process by which instances of weakening or projection may be commuted past instances of substitution in a derivation tree.

We conclude in Section 8 by showing that each of the monads constructed in the preceding sections has the good property of being local right adjoint. We show that the monads $W, P$ and $S$ for weakening, weakening and projection, and substitution, have the additional property of being cartesian, meaning that the naturality squares of their unit and multiplication are all pullbacks. We also discuss in more detail the further applications outlined above.

\section{Syntax OF TYPE THEORY}

2.1. Generalised algebraic theories. In this section, we give a careful exposition of the syntax of dependent type theory. As explained in the introduction, our concern is not with the type constructors of Martin-Löf type theory-identity types, $\Pi$-types, $\Sigma$-types, and so on-but rather with the basic structure of type dependency itself. It is thus a natural choice to work in the setting of Cartmell's generalised algebraic theories [5]; these are dependent sequent calculi without type constructors, but with the possibility of adding arbitrary (possibly dependent) type and term constants. To give such a theory is to give its type and term constants together with a list of axioms specifying the formation rules for the constants as well as any equational constraints they should satisfy.

Example 1. The generalised algebraic theory of categories is given over the language with two type-constants $O$ and $A$, two term-constants c and i, and the following axioms:

- $\vdash O$ type;

- $x: O, y: O \vdash A(x, y)$ type;

- $x: O \vdash \mathrm{i}(x): A(x, x)$;

- $x: O, y: O, z: O, g: A(y, z), f: A(x, y) \vdash \mathrm{c}(x, y, z, g, f): A(x, z)$;

- $x: O, y: O, f: A(x, y) \vdash \mathrm{c}(x, y, y, \mathrm{i}(y), f)=f: A(x, y)$;

- $x: O, y: O, f: A(x, y) \vdash \mathrm{c}(x, x, y, f, \mathrm{i}(x))=f: A(x, y)$;

- $x: O, y: O, z: O, w: O, h: A(z, w), g: A(y, z), f: A(x, y)$

$\vdash \mathrm{c}(x, y, w, \mathrm{c}(y, z, w, h, g), f)=\mathrm{c}(x, z, w, h, \mathrm{c}(x, y, z, g, f)): A(x, w)$.

We now give the formal definition; here, and throughout the paper, $V$ denotes a fixed denumerable set of variables.

Definition 2. (a) Given an alphabet $W$, the collection $W^{*}$ of expressions over $W$ is the smallest collection of strings closed under the rules:

- If $x \in V$ then $x \in W^{*}$;

- If $n \in \mathbb{N}, e_{1}, \ldots, e_{n} \in W^{*}$ and $w \in W$ then $w\left(e_{1}, \ldots, e_{n}\right) \in W^{*}$.

For the second clause, in the case $n=0$, we abbreviate $w()$ simply to $w$. We now define in the usual manner the free variables $\mathrm{fv}(e)$ of an expression, and the substitution $e^{\prime}[e / x]$ of an expression for a variable in an expression. 
(b) A context over the alphabet $W$ is a (possibly empty) string $x_{1}: T_{1}, \ldots, x_{n}$ : $T_{n}$, where each $x_{i}$ is a distinct element of $V$ and each $T_{i}$ is in $W^{*}$. A judgement over $W$ is a string taking one of the following four forms:

- A type judgement $\Gamma \vdash T$ type;

- A term judgement $\Gamma \vdash t: T$;

- A type equality judgement $\Gamma \vdash T=T^{\prime}$ type;

- A term equality judgement $\Gamma \vdash t=t^{\prime}: T$, where in each case $\Gamma$ is a context over $W$ and $t, t^{\prime}, T, T^{\prime} \in W^{*}$. The degree of a judgement is defined to be one greater than the length of its context.

(c) The boundary $\partial(\mathcal{J})$ of a judgement $\mathcal{J}$ over $W$ is a list of judgements of length 0,1 , or 2 , defined as follows:
- $\partial(\vdash T$ type $)=()$;
- $\partial\left(\Gamma, x: T \vdash T^{\prime}\right.$ type $)=(\Gamma \vdash T$ type $)$;
- $\partial(\Gamma \vdash t: T)=(\Gamma \vdash T$ type $)$;
- $\partial\left(\Gamma \vdash T=T^{\prime}\right.$ type $)=\left(\Gamma \vdash T\right.$ type, $\Gamma \vdash T^{\prime}$ type $)$;
- $\partial\left(\Gamma \vdash t=t^{\prime}: T\right)=\left(\Gamma \vdash t: T, \Gamma \vdash t^{\prime}: T\right)$.

(d) A collection $\Phi$ of judgements over $W$ is deductively closed if, whenever the hypotheses of one the rules in Table 1 is in $\Phi$, so too is the conclusion.

Definition 3. [5] A generalised algebraic theory (GAT) $\mathbb{T}$ comprises a collection $\Omega$ of type-constants, a collection $\Sigma$ of term-constants, and a collection $\Lambda$ of basic judgements over $\Omega \amalg \Sigma$ such that:

- For each $A \in \Omega$, there is a unique judgement in $\Lambda$ of the form

$$
x_{1}: T_{1}, \ldots, x_{n-1}: T_{n-1} \vdash A\left(x_{1}, \ldots, x_{n-1}\right) \text { type, }
$$

and we define the degree of $A$ to be the degree of this judgement;

- For each $f \in \Sigma$, there is a unique judgement in $\Lambda$ of the form

$$
x_{1}: T_{1}, \ldots, x_{n-1}: T_{n-1} \vdash f\left(x_{1}, \ldots, x_{n-1}\right): T,
$$

and again, we define the degree of $f$ to be the degree of this judgement;

- All other elements of $\Lambda$ are type equality or term equality judgements;

- Each element of $\Lambda$ is a derived judgement of $\mathbb{T}$.

Here, the collection $\mathbb{T}^{*}$ of derived judgements of $\mathbb{T}$ is the smallest deductively closed collection which contains a judgement $\mathcal{J} \in \Lambda$ whenever it contains each judgement in its boundary $\partial(\mathcal{J})$.

As we have said, the notion of GAT does not incorporate any of the usual typeforming operations of Martin-Löf type theory. To add these, we would extend the expression grammar of Definition 2 with syntax for the type-formers, and extend Table 1 with the corresponding formation, introduction, elimination and computation rules; see $[22,10]$ for detailed treatments in this spirit. In this paper, we are interested in understanding the interactions between the basic structural rules, and so, as anticipated in the introduction, we will find it more useful to vary 


$$
\begin{array}{ccc}
\frac{\Gamma \vdash t: T}{\Gamma \vdash t=t: T} & \frac{\Gamma \vdash t_{1}=t_{2}: T}{\Gamma \vdash t_{2}=t_{1}: T} & \frac{\Gamma \vdash t_{1}=t_{2}: T \quad \Gamma \vdash t_{2}=t_{3}: T}{\Gamma \vdash t_{1}=t_{3}: T} \\
\frac{\Gamma \vdash T \text { type }}{\Gamma \vdash T=T \text { type }} & \frac{\Gamma \vdash T_{1}=T_{2} \text { type }}{\Gamma \vdash T_{2}=T_{1}: T} & \frac{\Gamma \vdash T_{1}=T_{2} \text { type } \quad \Gamma \vdash T_{2}=T_{3} \text { type }}{\Gamma \vdash T_{1}=T_{3} \text { type }} \\
\frac{\Gamma \vdash T_{1}=T_{2} \text { type } \quad \Gamma \vdash t: T_{1}}{\Gamma \vdash t: T_{2}} & \frac{\Gamma \vdash T_{1}=T_{2} \text { type } \quad \Gamma \vdash t_{1}=t_{2}: T_{1}}{\Gamma \vdash t_{1}=t_{2}: T_{2}} \\
\frac{\Gamma \vdash \mathcal{J}}{\sigma \cdot \Gamma \vdash \sigma \cdot \mathcal{J}} & \sigma \in \operatorname{Sym}(V)
\end{array}
$$

(in the last rule, $\mathcal{J}$ denotes one of the four judgment types, and $\sigma \cdot \Gamma$ and $\sigma \cdot \mathcal{J}$ denote the action of the automorphism $\sigma$ of $V$ on the variables appearing in $\Gamma$ and $\mathcal{J}$.)

WEAKENING RULE

$$
\frac{\Gamma \vdash T \text { type } \quad \Gamma, \Delta \vdash \mathcal{J}}{\Gamma, y: T, \Delta \vdash \mathcal{J}} y \notin \mathrm{fv}(\Gamma) \cup \mathrm{fv}(\Delta)
$$

Projection RULE

$$
\frac{\Gamma \vdash T \text { type }}{\Gamma, y: T \vdash y: T} y \notin \mathrm{fv}(\Gamma)
$$

\section{Substitution RULES}

$$
\begin{gathered}
\frac{\Gamma \vdash t: T \quad \Gamma, y: T, \Delta \vdash \mathcal{J}}{\Gamma, \Delta[t / y] \vdash \mathcal{J}[t / y]} \\
\frac{\Gamma \vdash t_{1}=t_{2}: T \quad \Gamma, y: T, \Delta \vdash T^{\prime} \text { type }}{\Gamma, \Delta\left[t_{2} / y\right] \vdash T^{\prime}\left[t_{1} / y\right]=T^{\prime}\left[t_{2} / y\right] \text { type }} \\
\frac{\Gamma \vdash t_{1}=t_{2}: T \quad \Gamma, y: T, \Delta \vdash t^{\prime}: T^{\prime}}{\Gamma, \Delta\left[t_{2} / y\right] \vdash t^{\prime}\left[t_{1} / y\right]=t^{\prime}\left[t_{2} / y\right]: T^{\prime}\left[t_{2} / y\right]}
\end{gathered}
$$

TABLE 1. Deduction rules for generalised algebraic theories

the definition of GAT in the other direction, by removing some of the deduction rules.

Definition 4. Let $w, p$ and $s$ denote the weakening rules, the projection rules and the substitution rules, respectively. For any subset $D \subset\{w, p, s\}$, we define an "D-GAT" in the same manner as a GAT, but with the deduction rules of Table 1 reduced to those for equality, $\alpha$-conversion and the rules in $D$. Thus a GAT in the previous sense is equally a " $\{w, p, s\}$-GAT".

In fact, we should restrict this definition slightly. We call $D \subset\{w, p, s\}$ decent if it contains $w$ whenever it contains $p$; and in what follows, we consider only decent $D$. The reason for this restriction is that the projection rule requires the 
weakening rule to "make sense"; more precisely, the problem is that for indecent $D$, the $D$-GATS do not have the property that the boundary of a derivable judgement is again derivable. We exclude such pathologies by excluding such $D$.

2.2. Interpretations. The $(D-)$ GATs are the objects of a category, wherein morphisms are (equivalence-classes of) interpretations of one theory in another. In terms of this, we can, for example, define set-based models of a GAT $\mathbb{T}$ as interpretations of $\mathbb{T}$ in the "GAT of sets and families of sets", as described in $[5$, Section 14].

Definition 5. [5] An interpretation $\varphi: \mathbb{T} \rightarrow \mathbb{U}$ of (D-)GATs is given by an assignation $\varphi: \mathbb{T}^{*} \rightarrow \mathbb{U}^{*}$ on derived judgements such that:

- $\varphi$ preserves boundaries; thus $\varphi(\partial(\mathcal{J}))=\partial(\varphi(\mathcal{J}))$ for all $\mathcal{J} \in \mathbb{T}^{*}$.

- $\varphi$ preserves deduction in the following sense. To each deduction rule with $n$ premisses, we can associate an $n$-ary partial function $h$ from the judgements over a given alphabet to itself, sending the hypotheses of the rule to its conclusion. We now require that for each $\mathcal{J}_{1}, \ldots, \mathcal{J}_{n} \in \mathbb{T}^{*}$ we have $\varphi\left(h\left(\mathcal{J}_{1}, \ldots, \mathcal{J}_{n}\right)\right)=h\left(\varphi\left(\mathcal{J}_{1}\right), \ldots, \varphi\left(\mathcal{J}_{n}\right)\right)$ whenever both sides are defined. For example, preserving the first equality rule means that:

$$
\varphi(\Gamma \vdash T \text { type })=\left(\Gamma^{\prime} \vdash T^{\prime} \text { type }\right) \Longrightarrow \varphi(\Gamma \vdash T=T \text { type })=\left(\Gamma^{\prime} \vdash T^{\prime}=T^{\prime} \text { type }\right)
$$

while preserving the first substitution rule means that

$$
\begin{aligned}
\varphi(\Gamma \vdash t: T)=\left(\Gamma^{\prime} \vdash t^{\prime}:\right. & \left.T^{\prime}\right), \varphi(\Gamma, y: T, \Delta \vdash \mathcal{J})=\left(\Gamma^{\prime}, y: T^{\prime}, \Delta^{\prime} \vdash \mathcal{J}^{\prime}\right) \\
& \Longrightarrow \varphi(\Gamma, \Delta[t / y] \vdash \mathcal{J}[t / y])=\left(\Gamma^{\prime}, \Delta^{\prime}\left[t^{\prime} / y\right] \vdash \mathcal{J}^{\prime}\left[t^{\prime} / y\right]\right)
\end{aligned}
$$

Note that the requirement that an interpretation $\varphi$ preserve deductions means that it is uniquely determined by its action on basic judgements. More precisely, to specify an interpretation $\varphi$ it suffices to describe where each basic judgement $\mathcal{J}$ of $\mathbb{T}$ is sent, and to verify that these choices satisfy $\partial(\varphi(\mathcal{J}))=\varphi(\partial(\mathcal{J}))$; note that, since $\partial(\mathcal{J})$ is in general only a derived judgement of $\mathbb{T}$, the value $\varphi(\partial(\mathcal{J}))$ must be determined from the given values on basic judgements using the fact that $\varphi$ is required to preserve deduction.

As anticipated above, the morphisms of the category of GATs will not be interpretations, but rather equivalence classes of interpretations modulo the equivalence relation of provable equality, which we now define.

Definition 6. Let $\mathbb{T}$ be a $(D-)$ GAT. The congruence $\equiv$ on the derived type judgements of $\mathbb{T}$ is defined by asserting that

$$
\left(x_{1}: S_{1}, \ldots, x_{n-1}: S_{n-1} \vdash S_{n} \text { type }\right) \equiv\left(y_{1}: T_{1}, \ldots, y_{m-1}: T_{m-1} \vdash T_{m} \text { type }\right)
$$

if and only if $n=m$, and for each $1 \leqslant i \leqslant n$, the judgement

$$
x_{1}: S_{1}, \ldots, x_{i-1}: S_{i-1} \vdash S_{i}=T_{i}\left[x_{1} / y_{1}, \ldots, x_{i-1} / y_{i-1}\right] \text { type }
$$


is derivable. The congruence $\equiv$ on the derived term judgements of $\mathbb{T}$ is defined by asserting that

$$
\left(x_{1}: S_{1}, \ldots, x_{n-1}: S_{n-1} \vdash s: S_{n}\right) \equiv\left(y_{1}: T_{1}, \ldots, y_{m-1}: T_{m-1} \vdash t: T_{m}\right)
$$

if and only if their boundaries are congruent type judgements (in particular $n=m$ ) and moreover $\mathbb{T}$ derives that

$$
x_{1}: S_{1}, \ldots, x_{n-1}: S_{n-1} \vdash s=t\left[x_{1} / y_{1}, \ldots, x_{n-1} / y_{n-1}\right]: S_{n} .
$$

Definition 7. The category GAT has generalised algebraic theories as objects, and as morphisms, equivalence classes of interpretations $\mathbb{T} \rightarrow \mathbb{U}$, where two interpretations $\varphi$ and $\varphi^{\prime}$ are deemed equivalent just when $\varphi(\mathcal{J}) \equiv \varphi^{\prime}(\mathcal{J})$ for each derived type or term judgement of $\mathbb{T}$. We similarly define the category $D$-GAT for any decent $D \subset\{w, p, s\}$.

\section{TYPE-AND-TERM STRUCTURES AND MONADICITY}

3.1. Type-and-term structures. We now begin the main task of this paper, that of expressing the category GAT of generalised algebraic theories as the category of algebras for a monad on a presheaf category. As discussed in the introduction, the presheaf category at issue will model the collections of derived judgements of a type theory; more precisely, it will model the derivable type and term judgements considered modulo derivable equality.

Definition 8. Let $\mathcal{H}$ denote the category generated by the graph

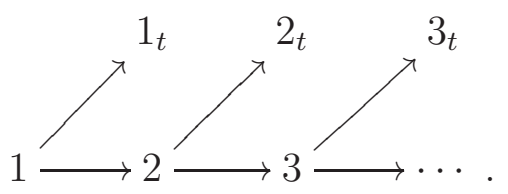

By a type-and-term structure, we mean a presheaf $X \in\left[\mathcal{H}^{\text {op }}\right.$, Set $]$. We write the reindexing maps $X\left(n_{t}\right) \rightarrow X(n)$ and $X(n+1) \rightarrow X(n)$ as $\partial$, and call them boundary maps. We refer to elements of $X(n)$ as type-elements of degree $n$, and elements of $X\left(n_{t}\right)$ as term-elements of degree $n$.

Remark 9. A type-and-term structure is exactly a computational arena in the sense of [12]. At the moment, this may appear to be a rather fanciful observation, but we will see in Remarks 18 and 24 below that it is part of a more substantial link with innocent game semantics.

The idea is that, for a type-and-term structure $X$, elements of $X(n)$ or of $X\left(n_{t}\right)$ should be thought of as 三-equivalence classes of type or term judgements of degree $n$ of a dependent sequent calcluls. The following definition makes this precise.

Definition 10. Let $D \subset\{w, p, s\}$ be decent. We define the forgetful functor $V=V_{D}: D$-GAT $\rightarrow\left[\mathcal{H}^{\mathrm{op}}\right.$, Set $]$ by sending an $A$-GAT $\mathbb{T}$ to the type-and-term 
structure $V \mathbb{T}$ with

$$
\begin{aligned}
V \mathbb{T}(n) & =\left\{\left(x_{1}: T_{1}, \ldots, x_{n-1}: T_{n-1} \vdash T_{n} \text { type }\right) \in \mathbb{T}^{*}\right\} / \equiv \\
\text { and } V \mathbb{T}\left(n_{t}\right) & =\left\{\left(x_{1}: T_{1}, \ldots, x_{n-1}: T_{n-1} \vdash t: T_{n}\right) \in \mathbb{T}^{*}\right\} / \equiv
\end{aligned}
$$

and with the maps $\partial: V \mathbb{T}(n+1) \rightarrow V \mathbb{T}(n)$ and $\partial: V \mathbb{T}\left(n_{t}\right) \rightarrow V \mathbb{T}(n)$ sending the equivalence class of a judgement to the equivalence class of its boundary. On maps, $V$ sends an interpretation $\varphi: \mathbb{T} \rightarrow \mathbb{U}$ to the presheaf map $V \varphi: V \mathbb{T} \rightarrow$ $V \mathbb{U}$ with $V \varphi([\mathcal{J}])=[\varphi(\mathcal{J})]$ (note this is well-defined as an interpretation must preserve boundaries and degrees).

In the analysis that follows, we will frequently find that most of the real action goes on at the level of type-elements, with the term-elements "coming along for the ride" in a fairly straightforward manner. In light of this, we will find convenient to introduce the following notation.

Definition 11. Given $X \in\left[\mathcal{H}^{\text {op }}\right.$, Set $]$ and $A \in X(n)$, we write $\operatorname{Tm}_{X}(A)$ for the set of all $a \in X\left(n_{t}\right)$ with $\partial a=A$; given $f: X \rightarrow Y$ in [ $\mathcal{H}^{\text {op }}$, Set], we write $f: \operatorname{Tm}_{X}(A) \rightarrow \operatorname{Tm}_{Y}(f(A))$ for the restriction of $f$ to such term-elements.

Now to specify $X \in\left[\mathcal{H}^{\text {op }}\right.$, Set $]$, it is enough to give sets of type-elements and boundary maps $X(1) \leftarrow X(2) \leftarrow X(3) \leftarrow \ldots$ together with sets $\operatorname{Tm}_{X}(A)$ of term-elements over each type-element $A$. Similarly, to given a map of presheaves $f: X \rightarrow Y$, it is enough to give maps $X(n) \rightarrow Y(n)$ for each $n>0$ and and maps $\operatorname{Tm}_{X}(A) \rightarrow \operatorname{Tm}_{Y}(f(A))$ for each $A \in X(n)$.

3.2. Monadicity. The following result now tells us that for each decent $D$, we can present $D$-GAT (to within equivalence) as the category of algebras for a monad on $\left[\mathcal{H}^{\text {op }}\right.$, Set $]$.

Proposition 12. For any decent $D$, the functor $V=V_{D}: D$-GAT $\rightarrow\left[\mathcal{H}^{\text {op }}\right.$, Set $]$ has a left adjoint and is monadic.

Proof. The left adjoint $G=G_{D}$ has value at a type-and-term structure $X$ given by the $D$-GAT $G X$ whose type-constants and term-constants are the respective type-elements and term-elements of $X$, and whose basic judgements are of the form

$$
\mathcal{J}_{A}=x_{1}: A_{1}, \ldots, x_{n-1}: A_{n-1}\left(x_{1}, \ldots, x_{n-2}\right) \vdash A\left(x_{1}, \ldots, x_{n-1}\right) \text { type }
$$

for each $A \in X(n)$ with successive boundaries $A_{n-1}, \ldots, A_{1}$, and

$$
\mathcal{J}_{a}=x_{1}: A_{1}, \ldots, x_{n-1}: A_{n-1}\left(x_{1}, \ldots, x_{n-2}\right) \vdash a\left(x_{1}, \ldots, x_{n-1}\right): A\left(x_{1}, \ldots, x_{n-1}\right)
$$

for each $a \in \operatorname{Tm}_{X}(A)$. The unit of the adjunction $\eta_{X}: X \rightarrow V G X$ is given by $\eta_{X}(A)=\left[\mathcal{J}_{A}\right]$ and $\eta_{X}(f)=\left[\mathcal{J}_{f}\right]$; the counit $\epsilon_{\mathbb{T}}: G V \mathbb{T} \rightarrow \mathbb{T}$ at an $D$-GAT $\mathbb{T}$ is the interpretation defined on basic judgements by $\epsilon_{X}\left(\mathcal{J}_{[\mathcal{J}]}\right)=\mathcal{J}$.

The monadicity of $V$ is verified by an application of Beck's theorem [19]. First note that $D$-GAT has all coequalisers: indeed, given interpretations $\varphi, \psi: \mathbb{T} \rightrightarrows$ $\mathbb{U}$, their coequaliser $\mathbb{U}^{\prime}$ is obtained from $\mathbb{U}$ by adjoining a basic equality judgement 
$\Gamma \vdash T=T^{\prime}$ type whenever $\varphi(\mathcal{J})=(\Gamma \vdash T$ type $)$ and $\psi(\mathcal{J})=\left(\Gamma^{\prime} \vdash T^{\prime}\right.$ type $)$ for some basic type judgement $\mathcal{J}$ of $\mathbb{T}$; and similarly adjoining a basic equality judgement $\Gamma \vdash t=t^{\prime}: T$ whenever $\varphi(\mathcal{J})=(\Gamma \vdash t: T)$ and $\psi(\mathcal{J})=\left(\Gamma^{\prime} \vdash t^{\prime}: T^{\prime}\right)$ for some basic term judgement $\mathcal{J}$ of $\mathbb{T}$. The evident interpretation $\mathbb{U} \rightarrow \mathbb{U}^{\prime}$ exhibits $\mathbb{U}^{\prime}$ as the coequaliser of $\varphi$ and $\psi$.

It is easy to see that $V$ reflects isomorphisms, and so to verify monadicity, it remains to show that $V$ preserves coequalisers of $V$-split coequaliser pairs. Let $\varphi, \psi: \mathbb{T} \rightrightarrows \mathbb{U}$ be interpretations, and let

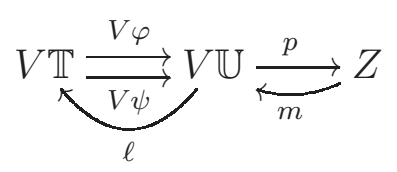

be a split coequaliser diagram in $\left[\mathcal{H}^{\text {op }}\right.$, Set $]$ : thus $p m=1, m p=V \psi \cdot \ell$ and $1=V \varphi \cdot \ell$, and these equations force $p$ to coequalise $V \varphi$ and $V \psi$. We must show that the coequaliser $q: \mathbb{U} \rightarrow \mathbb{U}^{\prime}$ of $\varphi$ and $\psi$ in GAT is preserved by $V$, i.e., that the comparison map $V q . m: Z \rightarrow V \mathbb{U}^{\prime}$ in $\left[\mathcal{H}^{\text {op }}\right.$, Set] is invertible. From the above explicit description of coequalisers in $D$-GAT, it is easy to see that the sets comprising $V \mathbb{U}^{\prime}$ are obtained by quotienting out those comprising $V \mathbb{U}$ by the smallest equivalence relation $\sim$ such that:

(a) $\varphi(\mathcal{J}) \sim \psi(\mathcal{J})$ for each basic type or term judgement of $\mathbb{T}$;

(b) $\mathcal{J}_{11} \sim \mathcal{J}_{12}, \ldots, \mathcal{J}_{k 1} \sim \mathcal{J}_{k 2}$ implies $\mathcal{J}_{1} \sim \mathcal{J}_{2}$ whenever one of the rules of Table 1 derives $\mathcal{J}_{i}$ from $\mathcal{J}_{1 i}, \ldots, \mathcal{J}_{k i}($ for $i=1,2)$.

To show that the comparison map $Z \rightarrow V \mathbb{U}^{\prime}$ is invertible is equally to show that $a \sim b$ implies $p(a)=p(b)$ for all $a, b$ in $V \mathbb{U}$. Clearly $p(\varphi(\mathcal{J}))=p(\psi(\mathcal{J}))$ for all basic type or term judgements of $\mathbb{T}$ since $p$ coequalises $V \varphi$ and $V \psi$; it remains to show that if that if $p\left(\mathcal{J}_{j 1}\right)=p\left(\mathcal{J}_{j 2}\right)$ in $V \mathbb{U}$ (for $j=1, \ldots, k$ ) and one of the rules of Table 1 derives $\mathcal{J}_{i}$ from $\mathcal{J}_{1 i}, \ldots, \mathcal{J}_{k i}$ (for $i=1,2$ ), then $p\left(\mathcal{J}_{1}\right)=$ $p\left(\mathcal{J}_{2}\right)$. As in Definition 5 , write $h$ for the $k$-ary partial function on judgements associated to the derivation rule at issue; thus we have $h\left(\mathcal{J}_{1 i}, \ldots, \mathcal{J}_{k i}\right)=\mathcal{J}_{i}$ (for $i=1,2)$. Examining the form of the rules in Table 1, we see that definedness of $h$ depends only on conditions involving boundaries; since $\ell: V \mathbb{U} \rightarrow V \mathbb{T}$ preserves boundaries, we conclude that $h\left(\ell\left(\mathcal{J}_{1 i}\right), \ldots, \ell\left(\mathcal{J}_{k i}\right)\right)$ is defined for $i=1,2$; and now

$$
\begin{aligned}
p\left(\mathcal{J}_{i}\right) & =p\left(h\left(\mathcal{J}_{1 i}, \ldots, \mathcal{J}_{k i}\right)\right)=p\left(h\left(\varphi \ell\left(\mathcal{J}_{1 i}\right), \ldots, \varphi \ell\left(\mathcal{J}_{k i}\right)\right)\right) \\
& =p\left(\varphi\left(h\left(\ell\left(\mathcal{J}_{1 i}\right), \ldots, \ell\left(\mathcal{J}_{k i}\right)\right)\right)=p\left(\psi\left(h\left(\ell\left(\mathcal{J}_{1 i}\right), \ldots, \ell\left(\mathcal{J}_{k i}\right)\right)\right)\right.\right. \\
& =p\left(h\left(\psi \ell\left(\mathcal{J}_{1 i}\right), \ldots, \psi \ell\left(\mathcal{J}_{k i}\right)\right)=p\left(h\left(m p\left(\mathcal{J}_{1 i}\right), \ldots, m p\left(\mathcal{J}_{k i}\right)\right)\right)\right.
\end{aligned}
$$

for $i=1,2$. But since $p\left(\mathcal{J}_{j 1}\right)=p\left(\mathcal{J}_{j 2}\right)$ for $j=1, \ldots, k$, we conclude that $p\left(\mathcal{J}_{1}\right)=p\left(\mathcal{J}_{2}\right)$ as required.

When $D=\emptyset$, we have a considerably stronger result: $V_{\emptyset}$ is an equivalence. This explains why have chosen to view GATs as monadic over $\left[\mathcal{H}^{\text {op }}\right.$, Set], rather than over some other presheaf category.

Proposition 13. $V_{\emptyset}: \emptyset-\mathrm{GAT} \rightarrow\left[\mathcal{H}^{\mathrm{op}}\right.$, Set $]$ is an equivalence of categories. 
Proof. We already know that $V_{\emptyset}$ has a left adjoint $G_{\emptyset}$ and is monadic, so it suffices to check that the unit $\eta: 1 \Rightarrow V_{\emptyset} G_{\emptyset}$ of the induced monad is invertible; thus, that for any $X \in\left[\mathcal{H}^{\text {op }}\right.$, Set $]$, each derived type or term judgement of $G_{\emptyset} X$ is 三-equivalent to a unique basic one. This follows by an easy induction on derivations.

In the following sections, we describe the monads $V_{D} G_{D}$ on [ $\mathcal{H}^{\mathrm{op}}$, Set] for various decent $A \subset\{w, p, s\}$. Our eventual objective is to do so in the case $A=\{w, p, s\}$, which we do in Section 7 below. We do this by way of various simpler cases: $A=\{w\}$ in Section $4, A=\{w, p\}$ in Section 5, and $A=\{s\}$ in Section 6. For the moment, let us record those aspects of the analysis common to all cases.

Proposition 14. For any decent $A \subset\{w, p, s\}$ and any $X \in\left[\mathcal{H}^{\mathrm{op}}\right.$, Set $]$, the only derivable type-equalities or term-equalities of the free $D$-GAT on $X$ are reflexivity judgements $\Gamma \vdash T=T$ type or $\Gamma \vdash t=t: T$. It follows that type- or termelements of $V_{D} G_{D}(X)$ are $\alpha$-equivalence classes of derivable judgements in the free $D$-GAT on $X$.

Proof. By induction on derivations.

\section{WEAKENING}

In this section, we describe the additional structure imposed on a type-andterm structure by the weakening rules; in other words, we will characterise the weakening monad $W$ induced by the free-forgetful adjunction $\{w\}$-GAT $\leftrightarrows$ $\left[\mathcal{H}^{\mathrm{op}}\right.$, Set $]$.

4.1. Underlying endofunctor. Our first step will be to describe the underlying endofunctor of the weakening monad. Its basic combinatorics are controlled by the notion of min-heap. Here, and throughout the rest of the paper, we use the notation $[a, b]$ to indicate the set of natural numbers $\{a, a+1, \ldots, b-1, b\}$, and write $[n]$ to mean $[1, n]$.

Definition 15. A min-heap of size $n$ is a function $\varphi:[0, n] \rightarrow[0, n]$ such that $\varphi(0)=0$ and $\varphi(i)<i$ for all $i \in[n]$. We write $\operatorname{Hp}(n)$ for the set of min-heaps of size $n$. For $\varphi \in \operatorname{Hp}(n+1)$, we write $\partial(\varphi)$ for $\left.\varphi\right|_{[0, n]} \in \operatorname{Hp}(n)$. For $\varphi \in \operatorname{Hp}(n)$ and $i \in[n]$, the depth of $i$ in $\varphi$ is defined to $\operatorname{bedp}_{\varphi}(i)=\left|\left\{\varphi(i), \varphi^{2}(i), \ldots\right\}\right|$.

To give $\varphi \in \operatorname{Hp}(n)$ is equally to give a partial order $\preccurlyeq \varphi$ on $[n]$ that is contained in the natural ordering ( $i \preccurlyeq_{\varphi} j$ implies $i \leqslant j$ ) and such that each downset $\downarrow i=\{j: j \preccurlyeq \varphi[i\}$ is a linear order. The partial order corresponding to a min-heap $\varphi$ is given by $i \preccurlyeq \varphi j$ iff $i=\varphi^{k}(j)$ for some $k$; conversely, the function associated to a partial order $\preccurlyeq$ is given by $\varphi(j)=\max \{i \in[0, j-1]: i=0$ or $i \preccurlyeq j\}$. In what follows, we will more frequently use the functional representation of min-heaps, but will switch to the relational representation where this is more convenient. 
We may depict $\varphi \in \operatorname{Hp}(n)$ by drawing the Hasse diagram of $\preccurlyeq \varphi$, which is a non-plane, directed forest with nodes $\{1, \ldots, n\}$, wherein the values labelling the nodes decrease along any directed path. For example:

$$
\begin{aligned}
& \varphi(1)=0 \quad \varphi(5)=2 \\
& \varphi(2)=1 \quad \varphi(6)=3 \\
& \varphi(3)=0 \quad \varphi(7)=1 \\
& \varphi(4)=2 \quad \varphi(8)=6
\end{aligned}
$$

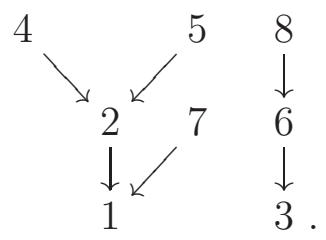

A forest of this kind with $n$ nodes can be seen as specifying the shape of a type judgement of degree $n$ in a free $\{w\}$-GAT; the numbers describe the ordering of types in the context, and the arrows indicate the dependencies between them. The following definition makes this precise.

Definition 16. Given $\varphi \in \operatorname{Hp}(n)$, we define the presheaf $[\varphi] \in\left[\mathcal{H}^{\mathrm{op}}\right.$, Set $]$ encoding a type judgement of shape $\varphi$ by:

$$
[\varphi](m)=\left\{i \in[n]: \operatorname{dp}_{\varphi}(i)=m\right\} \quad[\varphi]\left(m_{t}\right)=\emptyset
$$

with the non-trivial boundary maps $\partial:[\varphi](m+1) \rightarrow[\varphi](m)$ given by $i \mapsto \varphi(i)$.

So for any $X \in\left[\mathcal{H}^{\text {op }}\right.$, Set $]$, to give a map $h:[\varphi] \rightarrow X$ in $\left[\mathcal{H}^{\text {op }}\right.$, Set $]$ is to give

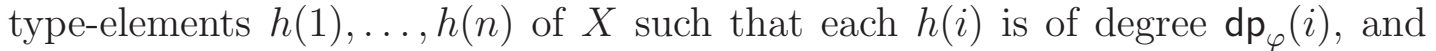
such that $\partial(h(i))=h(j)$ whenever $\varphi(i)=j$. If $n>1$, we write $\partial h$ for the restriction of $h$ along the evident inclusion $[\partial \varphi] \rightarrow[\varphi]$.

Proposition 17. The value at $X \in\left[\mathcal{H}^{\mathrm{op}}\right.$, Set $]$ of the underlying endofunctor of the weakening monad has type-elements and boundaries given by

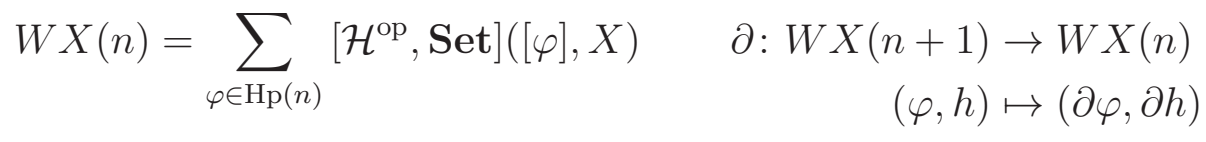

and term-elements $\operatorname{Tm}_{W X}(\varphi, h)=\operatorname{Tm}_{X}(h(n))$ for each $(\varphi, h) \in W X(n)$.

Proof. By Proposition 14, type-elements of $W X$ are $\alpha$-equivalence classes of type judgements in the free $\{w\}$-GAT on $X$. Each such equivalence class contains a unique judgement of the form

$$
\mathcal{J}=x_{1}: T_{1}, \ldots, x_{n-1}: T_{n-1} \vdash T_{n} \text { type } .
$$

Now by induction on derivations, we may show that each $T_{i}$ is of the form $A_{i}\left(x_{j_{1}}, \ldots, x_{j_{k-1}}\right)$ for some $A_{i} \in X(k)$ and $0<j_{1}<\cdots<j_{k-1}<i$. For each $i \in[n]$, we define $h(i)=A_{i}$, and $\varphi(i)=0$ if $k=1$ and $\varphi(i)=j_{k-1}$ otherwise. Taking also $\varphi(0)=0$, we obtain a min-heap $\varphi \in \operatorname{Hp}(n)$, and by a further induction on derivations, we see that

$$
\text { if } T_{i} \text { is } A\left(x_{j_{1}}, \ldots, x_{j_{k-1}}\right) \text { and } k>1 \text {, then } T_{j_{k-1}} \text { is }(\partial A)\left(x_{j_{1}}, \ldots, x_{j_{k-2}}\right) \text {, }
$$


which implies that $h$ is a well-defined map $[\varphi] \rightarrow X$. We thus have a function

$$
\begin{aligned}
\theta: W X(n) & \rightarrow \sum_{\varphi \in \operatorname{Hp}(n)}\left[\mathcal{H}^{\text {op }}, \operatorname{Set}\right]([\varphi], X) \\
{[\mathcal{J}] } & \mapsto\left(\varphi_{\mathcal{J}}, h_{\mathcal{J}}\right)
\end{aligned}
$$

Now (4.2) ensures that $\theta$ is injective, and it is clear that $\theta \partial=\partial \theta$. It remains to show surjectivity of $\theta$. Given a heap $\varphi \in \operatorname{Hp}(n)$ and $h:[\varphi] \rightarrow X$, we define a judgement $\mathcal{J}=\mathcal{J}(\varphi, h)$ as in (4.1) by taking each $T_{i}$ to be $h(i)\left(x_{j_{1}}, \ldots, x_{j_{k}}\right)$, where here $k=\operatorname{dp}_{\varphi}(i)$ and $j_{\ell}=\varphi^{k-\ell}(i)$ for $\ell \in[k-1]$. This $\mathcal{J}$ will then satisfy $\left(\varphi_{\mathcal{J}}, h_{\mathcal{J}}\right)=(\varphi, h)$ so long as it is in fact derivable in the free $\{w\}$-GAT on $X$.

We prove this by induction on $n$. The base case $n=1$ is clear; suppose then that $n>1$. If $\varphi(i)=i-1$ for all $i>0$, then $\mathcal{J}(\varphi, h)$ is a basic judgement, thus clearly derivable. Otherwise, there must exist some $m<n$ which is not in the image of $\varphi$ (in the corresponding forest, such an $m$ amounts to a leaf which is not the maximal node). Let $\left.\varphi\right|_{m}=\left.\varphi\right|_{[0, m]}$ in $\operatorname{Hp}(m)$, and let $\varphi \backslash m \in \operatorname{Hp}(n-1)$ be defined by

$$
(\varphi \backslash m)(i)= \begin{cases}\varphi(i) & i<m ; \\ \varphi(i+1) & i \geqslant m \text { and } \varphi(i+1)<m \\ \varphi(i+1)-1 & i \geqslant m \text { and } \varphi(i+1)>m\end{cases}
$$

(which amounts to stripping the leaf $m$ from the corresponding forest and renumbering appropriately). Let $\left.h\right|_{m}$ be the restriction of $h$ to $\left[\left.\varphi\right|_{m}\right]$, and let $h \backslash$ $m:[\varphi \backslash m] \rightarrow X$ take $i$ to $h(i)$ if $i<m$ and to $h(i+1)$ if $i \geqslant m$. By induction, $\mathcal{J}\left(\left.\varphi\right|_{m},\left.h\right|_{m}\right)$ and $\mathcal{J}(\varphi \backslash m, h \backslash m)$ are derivable judgements, and it is easy to see that, up to $\alpha$-equivalence, weakening $\mathcal{J}(\varphi \backslash m, h \backslash m)$ with respect to $\mathcal{J}\left(\left.\varphi\right|_{m},\left.h\right|_{m}\right)$ yields $\mathcal{J}(\varphi, h)$. Thus this latter judgement is derivable, and so the map (4.3) is indeed surjective.

This completes the proof on type judgements. It remains to consider term judgements. Any term judgement of the free $\{w\}$-GAT on $X$ is $\alpha$-equivalent to a unique one of the form

$$
x_{1}: T_{1}, \ldots, x_{n-1}: T_{n-1} \vdash t: T_{n} \text { type . }
$$

whose boundary is necessarily a type judgement $\mathcal{J}(\varphi, h)$ of the kind just described. By induction on derivations, we may show that if $T_{n}$ is $h(n)\left(x_{j_{1}}, \ldots, x_{j_{k-1}}\right)$ in (4.5), then $t$ is $a\left(x_{j_{1}}, \ldots, x_{j_{k-1}}\right)$ for some $a \in \operatorname{Tm}_{X}(h(n))$. We may thus identify $\operatorname{Tm}_{W X}(\varphi, h)$ with $\operatorname{Tm}_{X}(h(n))$, as required.

Remark 18. The above description of the endofunctor $W$ reveals it to be identical in form to one arising in the analysis of innocent game semantics given in [9]. Definition 10 of ibid. describes an exponential endofunctor ! (in fact a comonad) on a category Gam of games, whose meaning involves the addition of backtracking: thus, "weakening = backtracking". There are three main differences between our setting and that of [9], all relating to the category on which the endofunctor subsists. Modulo these three differences, the endofunctors are completely identical in form. 
- There are fewer objects in Gam than in $\left[\mathcal{H}^{\text {op }}\right.$, Set $]$ : games correspond to type-and-term structures without terms.

- There are more morphisms in the category of games; such morphisms correspond to certain kinds of (decorated) relations, rather than to functions.

- The category of games is polarised, in that elements at even and odd degrees act with opposite variances, and the endofunctor ! adds backtracking only at odd degrees. One way of understanding this polarisation is to observe that the "functional relations" $X \rightarrow Y$ in Gam are not maps of [ $\mathcal{H}^{\text {op }}$, Set]; rather, they correspond to diagrams

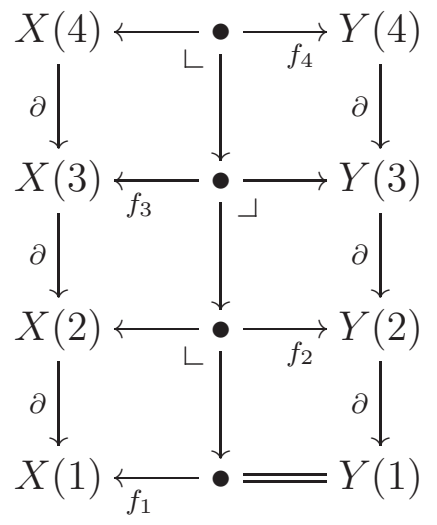

with all marked squares pullbacks. The underlying cause of these differences is as follows. In type theory, a context $(x: A, y: B(x), z: C(x, y), w$ : $D(x, y, z))$ is thought of as $\Sigma x: A . \Sigma y: B(x) . \Sigma z: C(x, y) . D(x, y, z)$. In game semantics, it would be interpreted as $\Pi x: A . \Sigma y: B(x)$. $z: C(x, y) . D(x, y, z)$.

4.2. Unit and multiplication. We now describe the unit $\eta^{W}: 1 \Rightarrow W$ and the multiplication $\mu^{W}: W W \Rightarrow W$ of the weakening monad. The unit is quite straightforward.

Definition 19. For any $n \geqslant 1$, we define $\gamma_{n} \in \operatorname{Hp}(n)$ by $\gamma_{n}(i)=0$ if $i=0$ and $\gamma_{n}(i)=i-1$ otherwise. For any $X \in\left[\mathcal{H}^{\text {op }}\right.$, Set $]$ and $A \in X(n)$, we define $\tilde{A}:\left[\gamma_{n}\right] \rightarrow X$ by $\tilde{A}(i)=\partial^{n-i}(A)$.

Note that $\preccurlyeq \gamma_{n}$ is just the usual linear ordering on $\{1, \ldots, n\}$. Moreover, $\left[\gamma_{n}\right]$ is isomorphic to the representable $\mathcal{H}(-, n)$, so that $\tilde{A}$ is simply the map corresponding to $A \in X(n)$ under the Yoneda lemma.

Proposition 20. For each $X \in\left[\mathcal{H}^{\text {op }}\right.$, Set $]$, the unit map $\eta_{X}^{W}: X \rightarrow W X$ of the weakening monad has components

$$
\begin{aligned}
X(n) & \rightarrow W X(n) & \operatorname{Tm}_{X}(A) & \rightarrow \operatorname{Tm}_{W X}\left(\gamma_{n}, \tilde{A}\right) \\
A & \mapsto\left(\gamma_{n}, \tilde{A}\right) & a & \mapsto a .
\end{aligned}
$$


Proof. The unit of the adjunction $G \dashv V$ at $X$ sends a type-element $A \in X(n)$ to the equivalence class of the basic judgement

$$
x_{1}: A_{1}, x_{2}: A_{2}\left(x_{1}\right), \ldots, x_{n-1}: A_{n-1}\left(x_{1}, \ldots, x_{n-2}\right) \vdash A\left(x_{1}, \ldots, x_{n-1}\right) \text { type } .
$$

Direct examination of the proof of Proposition 17 shows that this element is $\left(\gamma_{n}, \tilde{A}\right) \in W X(n)$. We argue similarly for term-elements.

We now describe the multiplication of $W$, which will be slightly more involved. First note that an element of $W^{2} X(n)$ is a pair $(\psi \in \operatorname{Hp}(n),(\varphi, h):[\psi] \rightarrow W X)$, where the second component picks out pairs $\left(\varphi_{i} \in \operatorname{Hp}\left(\operatorname{dp}_{\psi}(i)\right), h_{i}:\left[\varphi_{i}\right] \rightarrow X\right)$ for each $i \in[n]$ such that $\left(\partial \varphi_{i}, \partial h_{i}\right)=\left(\varphi_{j}, h_{j}\right)$ whenever $\psi(i)=j$. For such a type-element we have $\operatorname{Tm}_{W^{2} X}(\psi,(\varphi, h))=\operatorname{Tm}_{W X}\left(\varphi_{n}, h_{n}\right)=\operatorname{Tm}_{X}\left(h_{n}\left(\operatorname{dp}_{\psi}(n)\right)\right)$.

Definition 21. Given $(\psi,(\varphi, h)) \in W^{2} X(n)$, we define the heap $\psi \star \varphi \in \operatorname{Hp}(n)$ in relation form by

$$
i \preccurlyeq_{\psi \star \varphi} j \quad \text { iff } \quad i \preccurlyeq_{\psi} j \text { and } \# i \preccurlyeq_{\varphi_{j}} \# j
$$

or in function form by $(\psi \star \varphi)(i)=\psi^{\# i-\varphi_{i}(\# i)}(i)$; here, and elsewhere, we write $\# i$ as an abbreviation for $\mathrm{dp}_{\psi}(i)$. We define $\psi \star h:[\psi \star \varphi] \rightarrow X$ by $(\psi \star h)(i)=h_{i}(\# i)$. This is well-defined by the observation that if $(\psi \star \varphi)(i)=j$, then $\# j=\varphi_{i}(\# i)$, whence $\partial h_{i}(\# i)=h_{i}\left(\varphi_{i}(\# i)\right)=h_{i}(\# j)=h_{j}(\# j)$ (where the last equality holds since $\left.\partial h_{i}=h_{j}\right)$ as required.

Example 22. Suppose that $\psi \in \operatorname{Hp}(6)$ and $\varphi:[\psi] \rightarrow W 1$ are given by:

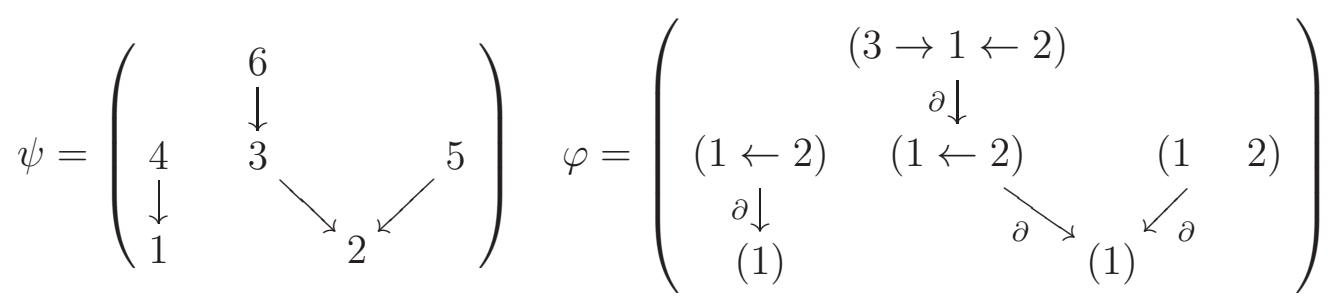

then $\psi \star \varphi$ is given by

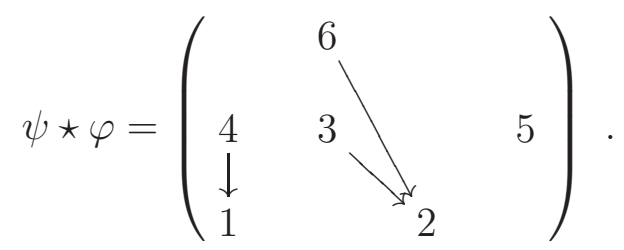

Proposition 23. For each $X \in\left[\mathcal{H}^{\mathrm{op}}\right.$, Set $]$, the multiplication $\mu_{X}^{W}: W^{2} X \rightarrow W X$ of the weakening monad has components

$$
\begin{aligned}
W^{2} X(n) & \rightarrow W X(n) & \operatorname{Tm}_{W^{2} X}(\psi,(\varphi, h)) & \rightarrow \operatorname{Tm}_{W X}(\psi \star \varphi, \psi \star h) \\
(\psi,(\varphi, h)) & \mapsto(\psi \star \varphi, \psi \star h) & a & \mapsto a .
\end{aligned}
$$

Proof. Let $(\psi, \ell)=(\psi,(\varphi, h)) \in W^{2} X(n)$. Note that if $\psi=\gamma_{n}$, then we have $\mu_{X}^{W}\left(\gamma_{n},(\varphi, h)\right)=\mu_{X}^{W}\left(\eta_{W X}^{W}\left(\varphi_{n}, h_{n}\right)\right)=\left(\varphi_{n}, h_{n}\right)$, and also by direct calculation that $\left(\gamma_{n} \star \varphi, \gamma_{n} \star h\right)=\left(\varphi_{n}, h_{n}\right)$. So without loss of generality we may assume 
$\psi \neq \gamma_{n}$. We proceed by induction on $n$. The base case $n=1$ is clear, since the only $\psi \in \operatorname{Hp}(1)$ is $\gamma_{1}$. So assume $n>1$ and $\psi \neq \gamma_{n}$. Then there exists some $m<n$ not in the image of $\psi$, and as in the proof of Proposition 17, we form $\left(\left.\psi\right|_{m},\left.\ell\right|_{m}\right) \in W^{2} X(m)$ and $(\psi \backslash m, \ell \backslash m) \in W^{2} X(n-1)$. By induction and a direct calculation, we have that

$$
\begin{aligned}
\mu_{X}^{W}\left(\left.\psi\right|_{m},\left.\ell\right|_{m}\right) & =\left(\left.\left.\psi\right|_{m} \star \varphi\right|_{m},\left.\left.\psi\right|_{m} \star h\right|_{m}\right)=\left(\left.(\psi \star \varphi)\right|_{m},\left.(\psi \star h)\right|_{m}\right) \\
\text { and } \mu_{X}^{W}(\psi \backslash m, \ell \backslash m) & =(\psi \backslash m \star \varphi \backslash m, \psi \backslash m \star h \backslash m)=((\psi \star \varphi) \backslash m,(\psi \star h) \backslash m) .
\end{aligned}
$$

Now the judgement $\mathcal{J}(\psi, \ell)$ is derivable by weakening $\mathcal{J}(\psi\rangle m, \ell m)$ with respect to $\mathcal{J}\left(\left.\psi\right|_{m},\left.\ell\right|_{m}\right)$ and $\alpha$-converting. Since $\mu_{X}^{W}$ is the image under the forgetful functor $V$ of the interpretation $\epsilon_{G X}: G V G X \rightarrow G X$, and interpretations preserve derivations, it follows that the judgement represented by $\mu_{X}^{W}(\psi, \ell)$ may be derived by weakening $\mathcal{J}((\psi \star \varphi) \backslash m,(\psi \star h) \backslash m)$ with respect to $\mathcal{J}\left(\left.(\psi \star \varphi)\right|_{m},\left.(\psi \star h)\right|_{m}\right)$ and $\alpha$-converting. But the judgement so obtained is easily seen to be $\mathcal{J}(\psi \star \varphi, \psi \star h)$, so that finally $\mu_{X}^{W}(\psi, \ell)=(\psi \star \varphi, \psi \star h)$ as required.

This completes the argument for type-elements. That for terms is similar; the key point is that if $a \in \operatorname{Tm}_{W^{2} X}(\psi, \ell)$ with $\psi \neq \gamma_{n}$, then on taking $m<n$ with $m \notin \operatorname{Im} \psi$ and forming $\left(\left.\psi\right|_{m},\left.\ell\right|_{m}\right) \in W^{2} X(m)$ and $(\psi \backslash m, \ell \backslash m) \in W^{2} X(n-1)$, we now have $a \in \operatorname{Tm}_{W^{2} X}(\psi \backslash m, \ell \backslash m)$. Weakening $\mathcal{J}(\psi \backslash m, \ell \backslash m, a)$ with respect to $\mathcal{J}\left(\left.\psi\right|_{m},\left.\ell\right|_{m}\right)$ yields back $\mathcal{J}(\psi, \ell, a)$, and we conclude the argument as before using induction and the preservation of derivations by $\mu_{X}^{W}$.

Remark 24. Again, we may link our monad $W$ to the exponential comonad! of [9]. The obvious difference is that one is a monad and the other a comonad. But this is easily accounted for due to the polarisation present in the category of games: the comonad ! corresponds to adding backtracking at "contravariant" odd degrees (there is a corresponding monad ? which adds backtracking at even degrees). The issue of polarity aside, the comonad structure of ! corresponds exactly to the monad structure of $W$ described above.

\section{WEAKENING AND PROJECTION}

We now turn to the additional structure imposed on a type-and-term structure by the projection rule. As we have already explained, the projection rule is not well-behaved in the absence of the weakening rule. Consequently, in this section, we will seek to characterise the weakening-and-projection monad $P$ induced by the free-forgetful adjunction $\{w, p\}$-GAT $\leftrightarrows\left[\mathcal{H}^{\mathrm{op}}\right.$, Set $]$.

5.1. Underlying endofunctor. As before, we begin by describing the underlying endofunctor of the weakening-and-projection monad.

Proposition 25. The value at $X \in\left[\mathcal{H}^{\mathrm{op}}\right.$, Set $]$ of the underlying endofunctor $P$ of the weakening-and-projection monad agrees with $W$ on type-elements, and on term-elements is given by

$$
\operatorname{Tm}_{P X}(\varphi, h)=\operatorname{Tm}_{X}(h(n))+\left\{\pi_{i}: i \in[n-1], \varphi(n)=\varphi(i), h(n)=h(i)\right\} .
$$


Note that the left-hand summand above is $\operatorname{Tm}_{W X}(\varphi, h)$, so that $P X$ is simply the extension of $W X$ by the addition of new term-elements representing projections. We write $\theta_{X}: W X \rightarrow P X$ for the evident inclusion maps.

Proof. By Proposition 14, elements of $P X$ are $\alpha$-equivalence classes of type or term judgements of the free $\{w, p\}$-GAT on $X$. The type judgements are visibly the same as those of the free $\{w\}$-GAT, while the term judgements augment those of the free $\{w\}$-GAT with ones of the form

$$
\mathcal{J}\left(\varphi, h, \pi_{i}\right)=x_{1}: T_{1}, \ldots, x_{n-1}: T_{n-1} \vdash x_{i}: T_{i}
$$

for $i \in[n-1]$ such that $T_{n}=T_{i}$; i.e., such that $\varphi(n)=\varphi(i)$ and $h(n)=h(i)$. This accounts for the right-hand summand in $\operatorname{Tm}_{P X}(\varphi, h)$.

5.2. Unit and multiplication. We now describe the unit $\eta^{P}: 1 \Rightarrow P$ and the multiplication $\mu^{P}: P P \Rightarrow P$ of the weakening-and-projection monad.

Proposition 26. For each $X \in\left[\mathcal{H}^{\text {op }}\right.$, Set $]$, the unit $\eta_{X}^{P}: X \rightarrow P X$ of the weakening-and-projection monad is the composite $\theta_{X} \circ \eta_{X}^{W}: X \rightarrow W X \rightarrow P X$.

Proof. Clear.

We now turn to the multiplication, for which we will need to identify termelements of $P^{2} X$ over a type-element $(\psi,(\varphi, h)) \in P^{2} X(n)=W^{2} X(n)$. By definition $\operatorname{Tm}_{P^{2} X}(\psi,(\varphi, n))$ is the set

$$
\operatorname{Tm}_{P X}\left(\varphi_{n}, h_{n}\right)+\left\{\pi_{i}(\psi,(\varphi, h)) \mid i \in[n-1], \psi(n)=\psi(i), \varphi_{n}=\varphi_{i}, h_{n}=h_{i}\right\}
$$

where we annotate the projections in the second factor to distinguish them from those appearing in the further decomposition of $\operatorname{Tm}_{P X}\left(\varphi_{n}, h_{n}\right)$ as

$$
\operatorname{Tm}_{X}\left(h_{n}(\# n)\right)+\left\{\pi_{i}\left(\varphi_{n}, h_{n}\right) \mid i \in[\# n-1], \varphi_{n}(\# n)=\varphi_{n}(i), h_{n}(\# n)=h_{n}(i)\right\} .
$$

Proposition 27. For each $X \in\left[\mathcal{H}^{\mathrm{op}}\right.$, Set $]$, the multiplication $\mu_{X}^{P}: P^{2} X \rightarrow P X$ of the weakening-and-projection monad agrees with that of $W$ on type-elements, and on term-elements is defined at $(\psi,(\varphi, n)) \in P^{2} X(n)$ to be the mapping $\operatorname{Tm}_{P^{2} X}(\psi,(\varphi, h)) \rightarrow \operatorname{Tm}_{P X}(\psi \star \varphi, \psi \star h)$ given by

$$
a \mapsto \begin{cases}a & \text { if } a \in \operatorname{Tm}_{X}\left(h_{n}(\# n)\right) ; \\ \pi_{i} & \text { if } a=\pi_{i}(\psi,(\varphi, h)) ; \\ \pi_{\psi^{\# n-i}(n)} & \text { if } a=\pi_{i}\left(\varphi_{n}, h_{n}\right) .\end{cases}
$$

Proof. The assertion concerning type-elements is clear. As for term-elements, an element of $\operatorname{Tm}_{P^{2} X}(\psi,(\varphi, h))$ that lies in $\operatorname{Tm}_{X}\left(h_{n}(\# n)\right)=\operatorname{Tm}_{W^{2} X}(\psi,(\varphi, h))$ represents a term judgement derived without the use of projection, so that the action of the multiplication is inherited from $W$. For an element of the form $\pi_{i}(\psi,(\varphi, h))$, the judgement of the free $\{w, p\}$-GAT on $W X$ which it represents is derivable from the judgement representing $(\psi,(\partial \varphi, \partial h))$ by a single application of the projection rule. As in the proof of Proposition 23, it follows that the judgement represented by $\mu_{X}^{P}\left(\pi_{i}(\psi,(\varphi, h))\right)$ is derivable from that representing 
$\mu_{X}^{P}(\psi,(\partial \varphi, \partial h))=(\partial(\psi \star \varphi), \partial(\psi \star h))$ by applying the same instance of the projection rule; it follows that $\mu_{X}^{P}\left(\pi_{i}(\psi,(\varphi, h))\right)=\pi_{i}$ as required.

Finally consider an element $\pi_{i}\left(\varphi_{n}, h_{n}\right) \in \operatorname{Tm}_{P^{2} X}(\psi,(\varphi, h))$. If $\psi=\gamma_{n}$, then $(\psi,(\varphi, h))=\eta_{P X}^{P}\left(\varphi_{n}, h_{n}\right)$ and the given term-element is the image under $\eta_{P X}^{P}$ of $\pi_{i} \in \operatorname{Tm}_{P X}\left(\varphi_{n}, h_{n}\right)$. It follows that applying $\mu_{X}^{P}$ yields back $\pi_{i}=\pi_{\gamma_{n}^{\# n-i}(n)}$, as required. For the case $\psi \neq \gamma_{n}$ we now proceed by induction on $n$; in what follows we abbreviate $\ell=(\varphi, h)$. The base case $n=1$ is trivial as then necessarily $\psi=\gamma_{1}$. So assume $n>1$ and $\psi \neq \gamma_{n}$. Then there is some $m<n$ not in the image of $\psi$, and as in the proofs of Propositions 17 and 23 we may form the type-elements $\left(\left.\psi\right|_{m},\left.\ell\right|_{m}\right)$ and $(\psi \backslash m, \ell \backslash m)$ and the term-element $\pi_{i}\left(\varphi_{n}, h_{n}\right) \in \operatorname{Tm}_{P^{2} X}(\psi \backslash m, \ell \backslash m)$. Now in the free $\{w, p\}$-GAT on $P X$, weakening the judgement represented by this term-element with respect to $\mathcal{J}\left(\left.\psi\right|_{m},\left.\ell\right|_{m}\right)$ yields the judgement representing $\pi_{i}\left(\varphi_{n}, h_{n}\right) \in \operatorname{Tm}_{P^{2} X}(\psi, \ell)$. As in the proof of Proposition 23, applying $\mu_{X}^{P}$ to $\left(\left.\psi\right|_{m},\left.\ell\right|_{m}\right)$ yields $\left(\left.(\psi \star \varphi)\right|_{m},\left.(\psi \star h)\right|_{m}\right)$, while by the inductive hypothesis, applying it to $\pi_{i}\left(\varphi_{n}, h_{n}\right) \in \operatorname{Tm}_{P^{2} X}(\psi \backslash m, \ell m)$ yields $\pi_{j} \in \operatorname{Tm}_{P X}((\psi \star \varphi) \backslash m,(\psi \star h) \backslash m)$ where here

$$
j=(\psi \backslash m)^{\mathrm{dp}_{\psi \backslash m}(n-1)-i}(n-1)=(\psi \backslash m)^{\# n-i}(n-1) .
$$

Since $m$ is not in the image of $\psi$, it follows easily from (4.4) that $j=\psi^{\# n-i}(n)$ if $i<m$ and $j=\psi^{\# n-i}(n)-1$ if $i>m$. Weakening the judgement represented by this term-element with respect to $\mathcal{J}\left(\left.(\psi \star \varphi)\right|_{m},\left.(\psi \star h)\right|_{m}\right)$ is easily seen (in either of the two cases $i<m$ and $i>m$ ) to yield the judgement represented by $\pi_{\psi \# n-i(n)}$, as required. We conclude as before by using the fact that $\mu_{X}^{P}$ preserves derivations.

\section{Substitution}

Our next step will be to consider the structure imposed on a type-and-term structure by the substitution rules, thus describing the substitution monad $S$ induced by the free-forgetful adjunction $\{s\}$-GAT $\leftrightarrows\left[\mathcal{H}^{\mathrm{op}}\right.$, Set $]$.

6.1. Underlying endofunctor. We begin by describing the underlying endofunctor of the substitution monad. Whereas the combinatorics of weakening are controlled by min-heaps, those of substitution are controlled by increasing lists of natural numbers.

Definition 28. A inc-list of length $n$ is a function $\alpha:[0, n] \rightarrow \mathbb{N}$ such that $\alpha(0)=0$ and $\alpha(i)>\alpha(j)$ whenever $i>j$. We write $\operatorname{Inc}(n)$ for the set of inc-lists of length $n$. Given $\alpha \in \operatorname{Inc}(n+1)$, we write $\partial(\alpha)$ for $\left.\alpha\right|_{[0, n]} \in \operatorname{Inc}(n)$.

An inc-list $\alpha$ can be seen as encoding the shape of a type judgement in a free $\{s\}$-GAT. The length of the list gives the degree of the judgement, while the values $\alpha(1), \ldots, \alpha(n)$ indicate the degrees of the individual types appearing in it. The case where $\alpha(i)=i$ for each $i$ encodes a judgement without substitution; otherwise, we have values $\alpha(m)$ and $\alpha(m+1)$ that are not consecutive, and this must be compensated for by the substitution of suitable terms into the $m+1$ st 
type to reduce its degree to merely one greater than that of the $m$ th type. The following definition make this precise.

Definition 29. Given $\alpha \in \operatorname{Inc}(n)$, we define the presheaf $[\alpha] \in\left[\mathcal{H}^{\text {op }}\right.$, Set $]$ encoding a type judgement of shape $\alpha$ by:

$$
[\alpha](i)=\left\{\begin{array}{ll}
\{i\} & \text { if } i \leqslant \alpha(n) ; \\
\emptyset & \text { otherwise; }
\end{array} \quad \text { and }[\alpha]\left(i_{t}\right)= \begin{cases}\left\{i_{t}\right\} & \text { if } i \leqslant \alpha(n), i \notin \operatorname{Im} \alpha \\
\emptyset & \text { otherwise. }\end{cases}\right.
$$

with the unique possible boundary maps.

For example, if $\alpha \in \operatorname{Inc}(3)$ has values $0<2<3<5<8$, then $[\alpha]$ is given by:

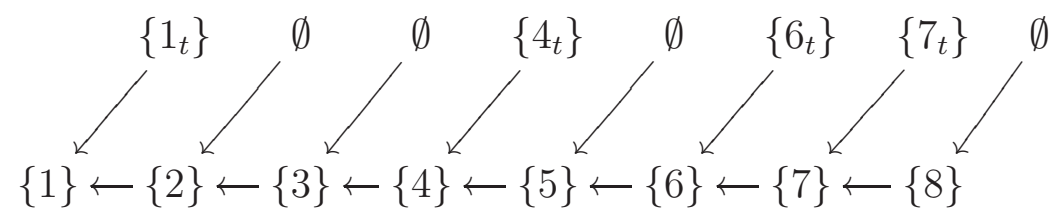

For a general $\alpha \in \operatorname{Inc}(n)$, a map $h:[\alpha] \rightarrow X$ in $\left[\mathcal{H}^{\text {op }}\right.$, Set $]$ is determined by giving, firstly, a type-element $h(\alpha(n)) \in X(\alpha(n))$-which determines $h(i) \in X(i)$ for each smaller $i$ by taking iterated boundaries - and secondly, term-elements $h\left(i_{t}\right) \in \operatorname{Tm}_{X}(h(i))$ for each $i \in[\alpha(n)] \backslash \operatorname{Im} \alpha$. When $n>1$, we write $\partial h:[\partial \alpha] \rightarrow X$ for the restriction of $h$ along the obvious inclusion $[\partial \alpha] \rightarrow[\alpha]$.

Proposition 30. The value at $X \in\left[\mathcal{H}^{\text {op }}\right.$, Set $]$ of the underlying endofunctor of the substitution monad has type-elements and boundaries given by

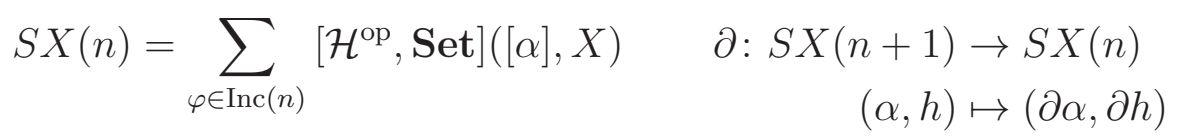

and term-elements $\operatorname{Tm}_{S X}(\alpha, h)=\operatorname{Tm}_{X}(h(\alpha(n)))$ for each $(\alpha, h) \in S X(n)$.

Proof. We prove by induction on derivations that, if $\mathcal{J}$ is a representative type judgement (4.1) of the free $\{s\}$-GAT on $X$, then there are natural numbers $0<$ $\alpha(1)<\cdots<\alpha(n)$, type-elements $A_{i} \in X(\alpha(i))$ for $i \in[n]$, and term-elements $a_{i} \in X\left(i_{t}\right)$ for $i \in[\alpha(n)] \backslash \operatorname{Im} \alpha$, such that each $T_{i}$ in $\mathcal{J}$ is of the form

$$
A_{i}\left(x_{1}, \ldots, x_{\alpha(i)-1}\right)\left[t_{\alpha(i)-1} / x_{\alpha(i)-1}\right] \ldots\left[t_{1} / x_{1}\right]\left[x_{1} / x_{\alpha(1)}\right] \ldots\left[x_{i-1} / x_{\alpha(i-1)}\right] ;
$$

here, $t_{i}$ is the expression $x_{i}$ if $i \in \operatorname{Im} \alpha$ and is $a_{i}\left(x_{1}, \ldots, x_{i-1}\right)$ otherwise. By a further induction on derivations, we may show that each $A_{i}$ and each $\partial\left(a_{i}\right)$ is of the form $\partial^{\ell}\left(A_{n}\right)$ for a suitable $\ell$. It follows that we have a well-defined map $h:[\alpha] \rightarrow X$ given by $h(i)=\partial^{\alpha(n)-i}\left(A_{n}\right)$ and $h\left(i_{t}\right)=a_{i}$; and that the pair $(\alpha, h)$ encodes all the information of the type judgement $\mathcal{J}$.

A similar induction on derivations shows that a term judgement $\mathcal{J}^{\prime}$ of the form (4.5) in the free $\{s\}$-GAT on $X$ is given by a type judgement as above together with a term expression $t$ of the form

$$
a\left(x_{1}, \ldots, x_{\alpha(n)-1}\right)\left[t_{\alpha(n)-1} / x_{\alpha(n)-1}\right] \ldots\left[t_{1} / x_{1}\right]\left[x_{1} / x_{\alpha(1)}\right] \ldots\left[x_{i-1} / x_{\alpha(n-1)}\right]
$$


for some $a \in \operatorname{Tm}_{X}\left(A_{n}\right)=\operatorname{Tm}_{X}(h(\alpha(n)))$. We thus have maps

$$
\begin{aligned}
& S X(n) \rightarrow \sum_{\alpha \in \operatorname{Inc}(n)}\left[\mathcal{H}^{\mathrm{op}}, \operatorname{Set}\right]([\alpha], X) \quad \operatorname{Tm}_{S X}([\mathcal{J}]) \rightarrow \operatorname{Tm}_{X}(h(\alpha(n))) \\
& {[\mathcal{J}] \mapsto\left(\alpha_{\mathcal{J}}, h_{\mathcal{J}}\right) \quad\left[\mathcal{J}^{\prime}\right] \mapsto a_{\mathcal{J}^{\prime}}}
\end{aligned}
$$

which by the above are well-defined, injective and compatible with boundaries. It remains to prove their surjectivity. Given $\alpha \in \operatorname{Inc}(n)$ and $h:[\alpha] \rightarrow X$, by taking $a_{i}=h\left(i_{t}\right)$ (for $i \in[\alpha(n)] \backslash \operatorname{Im} \alpha$ ) and defining $T_{i}$ as in (6.1), we obtain a type judgement $\mathcal{J}(\alpha, h)$ of the form (4.1), whose image under (6.2) will be $(\alpha, h)$ so long as it is in fact a derivable type judgement of the free $\{s\}$-GAT on $X$. Similarly, to any $a \in \operatorname{Tm}_{X}(h(\alpha(n)))$ we may assign a term judgement $\mathcal{J}(\alpha, h, a)$ with boundary $\mathcal{J}(\alpha, h)$ which will be sent to $a$ by the right-hand map in (6.2) so long as it is in fact derivable.

We prove derivability of these two kinds of judgements simultaneously by induction on the value $\alpha(n)-n$. In the base case $\alpha(n)=n$ we see easily that $\mathcal{J}(\alpha, h)$ and $\mathcal{J}(\alpha, h, a)$ are basic judgements of the free $\{s\}$-GAT on $X$, and so derivable. For the inductive step, suppose that $\alpha(n)-n>0$, and we wish to derive $\mathcal{J}(\alpha, h)$. Choose some $m<n$ and $j \in \mathbb{N}$ such that $\alpha(m)<j<\alpha(m+1)$. We now define $\alpha_{j} \in \operatorname{Inc}(n+1)$ and $\alpha^{j} \in \operatorname{Inc}(m+1)$ by

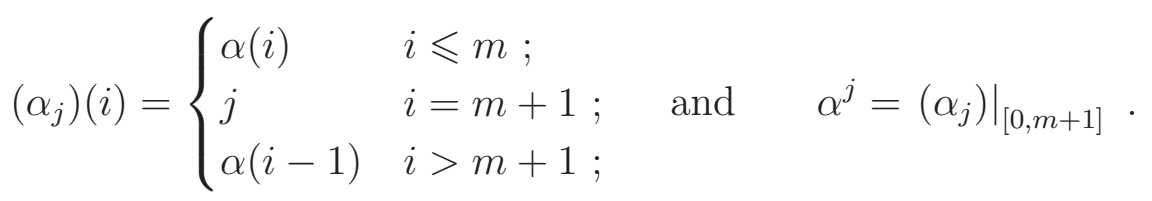

Note that $\alpha_{j}(n+1)=\alpha(n)$ since $n+1>m+1$, and so $\alpha_{j}(n+1)-(n+1)<$ $\alpha(n)-n$. Likewise $\alpha^{j}(m+1)=j<\alpha(m+1)$ and so $\alpha^{j}(m+1)-(m+1)<$ $\alpha(m+1)-(m+1) \leqslant \alpha(n)-n$. Note further that $\left[\alpha_{j}\right]$ and $\left[\alpha^{j}\right]$ are subpresheaves of $[\alpha]$; we write $h_{j}$ and $h^{j}$ for the restrictions of $h$ to them. By induction, $\mathcal{J}\left(\alpha_{j}, h_{j}\right)$ is a derivable type judgement and $\mathcal{J}\left(\alpha^{j}, h^{j}, h\left(j_{t}\right)\right)$ a derivable term judgement, and substituting the latter into the former (and $\alpha$-converting) now yields the required derivation of $\mathcal{J}(\alpha, h)$. In a similar manner, each term judgement $\mathcal{J}(\alpha, h, a)$ may be derived inductively from $\mathcal{J}\left(\alpha_{j}, h_{j}, a\right)$ and $\mathcal{J}\left(\alpha^{j}, h^{j}, h\left(j_{t}\right)\right)$.

6.2. Unit and multiplication. We now describe the unit $\eta^{S}: 1 \Rightarrow S$ and the multiplication $\mu^{S}: S S \Rightarrow S$ of the substitution monad.

Definition 31. For any $n \geqslant 1$, we write $\iota_{n} \in \operatorname{Inc}(n)$ for the inc-list given by $\iota_{n}(i)=i$. For any $X \in\left[\mathcal{H}^{\text {op }}\right.$, Set $]$ and $A \in X(n)$, we define $\tilde{A}:\left[\iota_{n}\right] \rightarrow X$ by $\tilde{A}(i)=\partial^{n-i}(A)$ for each $i \in[n]$.

As before, $\left[\iota_{n}\right]$ is isomorphic to the representable $\mathcal{H}(-, n)$ so that $\tilde{A}$ corresponds to $A \in X(n)$ under the Yoneda lemma. 
Proposition 32. For each $X \in\left[\mathcal{H}^{\text {op }}\right.$, Set $]$, the unit map $\eta_{X}^{S}: X \rightarrow S X$ of the substitution monad has components

$$
\begin{aligned}
X(n) & \rightarrow S X(n) & \operatorname{Tm}_{X}(A) & \rightarrow \operatorname{Tm}_{S X}\left(\iota_{n}, \tilde{A}\right) \\
A & \mapsto\left(\iota_{n}, \tilde{A}\right) & a & \mapsto a .
\end{aligned}
$$

Proof. Immediate from examination of the proof of Proposition 30.

We now turn to the multiplication of $S$, for which we need an explicit description of $S^{2} X$. Given $\alpha \in \operatorname{Inc}(n)$, a map $[\alpha] \rightarrow S X$ is determined as in the discussion following Definition 29 by its value at $\alpha(n)$ and its values at $i_{t}$ for each $i \in[\alpha(n)] \backslash \operatorname{Im} \alpha$. Giving these data amounts to giving

- An element $(\beta \in \operatorname{Inc}(\alpha(n)), h:[\beta] \rightarrow X) \in S X(\alpha(n))$; and

- Elements $k(i) \in \operatorname{Tm}_{S X}\left(\partial^{\alpha(n)-i}(\beta, h)\right)=\operatorname{Tm}_{X}(h(\beta(i)))$ for $i \in[\alpha(n)] \backslash \operatorname{Im} \alpha$.

Thus we write a typical type-element of $S^{2} X$ as $(\alpha \in \operatorname{Inc}(n),(\beta, h, k):[\alpha] \rightarrow S X)$. Now on terms, we have $\operatorname{Tm}_{S^{2} X}(\alpha,(\beta, h, k))=\operatorname{Tm}_{S X}(\beta, h)=\operatorname{Tm}_{X}(h(\beta(\alpha(n))))$.

Definition 33. Given $(\alpha,(\beta, h, k)) \in S^{2} X(n)$, we define the inc-list $\beta \alpha \in \operatorname{Inc}(n)$ by $(\beta \alpha)(i)=\beta(\alpha(i))$ and define $h \cup k:[\beta \alpha] \rightarrow X$ by taking $(h \cup k)(i)=h(i)$ for $i \in[\beta \alpha(n)]$ and for $i \in[\beta \alpha(n)] \backslash \operatorname{Im} \beta \alpha$ taking

$$
(h \cup k)\left(i_{t}\right)= \begin{cases}h\left(i_{t}\right) & \text { for } i \notin \operatorname{Im} \beta ; \\ k(j) & \text { for } i=\beta(j), j \notin \operatorname{Im} \alpha .\end{cases}
$$

Proposition 34. For each $X \in\left[\mathcal{H}^{\text {op }}\right.$, Set $]$, the multiplication $\mu_{X}^{S}: S^{2} X \rightarrow S X$ of the substitution monad has components

$$
\begin{aligned}
S^{2} X(n) & \rightarrow S X(n) & \operatorname{Tm}_{S^{2} X}(\alpha,(\beta, h, k)) & \rightarrow \operatorname{Tm}_{S X}(\beta \alpha, h \cup k) \\
(\alpha,(\beta, h, k)) & \mapsto(\beta \alpha, h \cup k) & a & \mapsto a .
\end{aligned}
$$

Proof. We prove the result for type- and term-elements simultaneously by induction on $\alpha(n)-n$. Consider first a type-element $(\alpha, \ell)=(\alpha,(\beta, h, k)) \in S^{2} X(n)$. In the base case where $\alpha(n)=n$, we must have $\alpha=\iota_{n}$ and now $\mu_{X}^{S}\left(\iota_{n},(\beta, h, k)\right)=$ $\mu_{X}^{S}\left(\eta_{S X}^{S}(\beta, h)\right)=(\beta, h)$, which is visibly equal to $\left(\beta \iota_{n}, h \cup k\right)$ (since in this case $k$ is trivial). For the inductive step, assume $\alpha(n)>n$. As in the proof of Proposition 30, we can find some $m<n$ and some $j \in \mathbb{N}$ with $\alpha(m)<j<\alpha(m+1)$ and now form the type-elements $\left(\alpha_{j}, \ell_{j}\right) \in S^{2} X(n+1)$ and $\left(\alpha^{j}, \ell^{j}\right) \in S^{2} X(m+1)$ and term-element $\ell\left(j_{t}\right)=k(j) \in \operatorname{Tm}_{S^{2} X}\left(\alpha^{j}, \ell^{j}\right)$.

Now $\ell_{j}=\left(\beta, h, k^{\prime}\right):\left[\alpha_{j}\right] \rightarrow X$, where $k^{\prime}$ is obtained from $k$ by removing the value at $j$; while $\ell^{j}=\left(\left.\beta\right|_{[0, j]}, h^{\prime}, k^{\prime \prime}\right)$, where where $h^{\prime}$ is the restriction of $h$ along the inclusion $\left[\left.\beta\right|_{[0, j]}\right] \rightarrow[\beta]$ and $k^{\prime \prime}$ is the restriction of $k$ to $[j-1]$. Thus by induction and direct calculation, we see that

$$
\begin{gathered}
\mu_{X}^{S}\left(\alpha_{j}, \ell_{j}\right)=\left(\beta \alpha_{j}, h \cup k^{\prime}\right)=\left((\beta \alpha)_{\beta(j)},(h \cup k)_{\beta(j)}\right) \\
\text { and } \mu_{X}^{S}\left(\alpha^{j}, \ell^{j}\right)=\left(\left(\left.\beta\right|_{[0, j]}\right) \alpha^{j}, h^{\prime} \cup k^{\prime \prime}\right)=\left((\beta \alpha)^{\beta(j)},(h \cup k)^{\beta(j)}\right),
\end{gathered}
$$


and that $k(j) \in \operatorname{Tm}_{S^{2} X}\left(\alpha^{j}, \ell^{j}\right)$ is sent to $k(j) \in \operatorname{Tm}_{S X}\left((\beta \alpha)^{\beta(j)},(h \cup k)^{\beta(j)}\right)$. Now the judgement $\mathcal{J}(\alpha, \ell)$ of the free $\{s\}$-GAT on $S X$ is derivable by substituting $\mathcal{J}\left(\alpha^{j}, \ell^{j}, k(j)\right)$ into $\mathcal{J}\left(\alpha_{j}, \ell_{j}\right)$ and $\alpha$-converting, and it follows that the judgement represented by $\mu_{X}^{S}(\alpha, \ell)$ may be derived by substituting $\mathcal{J}\left((\beta \alpha)^{\beta(j)},(h \cup\right.$ $\left.k)^{\beta(j)}, k(j)\right)$ into $\mathcal{J}\left((\beta \alpha)_{\beta(j)},(h \cup k)_{\beta(j)}\right)$ and $\alpha$-converting; whence $\mu_{X}^{S}(\alpha,(\beta, h, k))=$ $(\beta \alpha, h \cup k)$ as required. The argument for term judgements is similar, and hence omitted.

\section{Combining the structures}

We now combine the results of the preceding three sections in order to describe the structure imposed on a type-and-term structure by all the deduction rules of generalised algebraic theories; we will thus describe the monad $T$ for GATs induced by the free-forgetful adjunction GAT $\leftrightarrows\left[\mathcal{H}^{\mathrm{op}}\right.$, Set $]$.

Let $P$ and $S$ denote, as in the preceding sections, the weakening-and-projection monad and the substitution monad on $\left[\mathcal{H}^{\text {op }}\right.$, Set $]$. We have natural transformations $\rho: P \Rightarrow T \Leftarrow S: \sigma$ expressing that every derivable judgement of the free $\{w, p\}$ - or $\{s\}$-GAT on some $X$ is also derivable in the free GAT on $X$. It is easy to see that $\rho$ and $\sigma$ are compatible with the unit and multiplication maps and so exhibit $P$ and $S$ as submonads of $T$. Our task in this section will be to describe how these submonads combine together to yield $T$.

7.1. Underlying endofunctor. We first characterise the underlying endofunctor of the monad $T$ for GATs in terms of those of the weakening-and-projection and substitution monads. Our result expresses that every judgement of the free GAT on $X$ may be obtained in a unique way (up to $\alpha$-conversion) by first applying substitution to basic judgements, and then weakening and projection to these substituted judgements.

Proposition 35. For any $X \in\left[\mathcal{H}^{\text {op }}\right.$, Set $]$ the composite map

$$
\kappa_{X}:=P S X \stackrel{P \tau_{X}}{\longrightarrow} P T X \stackrel{\sigma_{T X}}{\longrightarrow} T T X \stackrel{\mu_{X}^{T}}{\longrightarrow} T X
$$

is invertible.

Note first that a type-element of $P S X$ has the form $(\varphi,(\alpha, h))$, where $\varphi \in$ $\operatorname{Hp}(n)$ and $\left(\alpha_{i}, h_{i}\right) \in S X(\# i)$ for each $i \in[n]$, such that $\left(\partial \alpha_{i}, \partial h_{i}\right)=\left(\alpha_{j}, h_{j}\right)$ whenever $\varphi(i)=j$. Furthermore, $\operatorname{Tm}_{P S X}(\varphi,(\alpha, h))$ is given by the sum

$$
\operatorname{Tm}_{S X}\left(\alpha_{n}, h_{n}\right)+\left\{\pi_{i} \mid i \in[n-1], \varphi(n)=\varphi(i), \alpha_{n}=\alpha_{i}, h_{n}=h_{i}\right\} .
$$

Proof. Consider first a representative type judgement $\mathcal{J}$ of the form (4.1) in the free GAT on $X$. By induction on derivations, we show that for each $i \in[n]$ there are $0<j_{1}<\cdots<j_{k-1}<i$ such that $\mathrm{fv}\left(T_{i}\right)=\left\{x_{j_{1}}, \ldots, x_{j_{k-1}}\right\}$ and such that

$$
x_{j_{1}}: T_{j_{1}}, \ldots, x_{j_{k-1}}: T_{j_{k-1}} \vdash T_{i} \text { type }
$$

is derivable in the free $\{s\}$-GAT on $X$. Define $\varphi(i)$ to be 0 if $k=1$ and to be $j_{k-1}$ otherwise, and define $\left(\alpha_{i}, h_{i}\right) \in S X(k)$ to be the element representing the 
$\alpha$-equivalence class of (7.3). Taking also $\varphi(0)=0$ we obtain a heap $\varphi \in \operatorname{Hp}(n)$; moreover, for those $i$ with $\varphi(i)>0$ we see by a further induction on derivations that $\mathrm{fv}\left(T_{j_{k-1}}\right)=\left\{x_{j_{1}}, \ldots, x_{j_{k-2}}\right\}$, whence $\partial\left(\alpha_{i}, h_{i}\right)=\left(\alpha_{\varphi(i)}, h_{\varphi(i)}\right)$. Thus we have a well-defined map $(\alpha, h):[\varphi] \rightarrow S X$ and so an element $(\varphi,(\alpha, h)) \in P S X(n)$. In this way, we have defined a mapping

$$
\begin{aligned}
\theta: T X(n) & \rightarrow P S X(n) \\
{[\mathcal{J}] } & \mapsto\left(\varphi_{\mathcal{J}},\left(\alpha_{\mathcal{J}}, h_{\mathcal{J}}\right)\right)
\end{aligned}
$$

which we claim is inverse to the $n$-component of (7.1). It is easy to see that $\theta$ is injective, so it is enough to show that $1=\theta \circ \kappa_{X}: P S X(n) \rightarrow T X(n) \rightarrow P S X(n)$.

So let $(\varphi, \ell)=(\varphi,(\alpha, h)) \in P S X(n)$. If $\varphi=\gamma_{n}$, then $(\varphi, \ell)=\eta_{S X}^{P}\left(\alpha_{n}, h_{n}\right)$. Now by direct calculation $\kappa \circ \eta^{P} S=\tau$ so that $\kappa_{X}(\varphi, \ell)=\tau_{X}\left(\alpha_{n}, h_{n}\right)$ represents the judgement $\mathcal{J}\left(\alpha_{n}, h_{n}\right)$ of the free GAT on $X$. But by inspection, the image of $\mathcal{J}\left(\alpha_{n}, h_{n}\right)$ under $\theta$ is again $\left(\gamma_{n},(\alpha, h)\right)$, as required. For the case $\varphi \neq \gamma_{n}$ we proceed by induction on $n$. The case $n=1$ is trivial; so assume $n>1$. As in the proof of Proposition 17, we may find $m<n$ such that $m \notin \operatorname{Im} \varphi$ and form the type-elements $\left(\left.\varphi\right|_{m},\left.\ell\right|_{m}\right)$ and $(\varphi \backslash m, \ell m)$ of $P S X$. Now the judgement $\mathcal{J}(\varphi, \ell)$ of the free $\{w, p\}$-GAT on $S X$ is derivable by weakening $\mathcal{J}(\varphi \backslash m, \ell \backslash m)$ with respect to $\mathcal{J}\left(\left.\varphi\right|_{m},\left.\ell\right|_{m}\right)$ and $\alpha$-converting. It follows that the judgement represented by $\kappa_{X}(\varphi, \ell)$ is obtained in the same way from the judgements represented by $\kappa_{X}\left(\left.\varphi\right|_{m},\left.\ell\right|_{m}\right)$ and $\kappa_{X}(\varphi \backslash m, \ell \backslash m)$. But by induction, these latter judgements are sent to $\left(\left.\varphi\right|_{m},\left.\ell\right|_{m}\right)$ and $(\varphi \backslash m, \ell \backslash m)$ by $\theta$, and now by direct inspection of the description of $\theta$ given above, we conclude that $\theta\left(\kappa_{X}(\varphi, \ell)\right)=(\varphi, \ell)$, as required.

This completes the argument on type judgements; that on term judgements is similar. The key point is that we may show by induction on derivations that a typical term judgement $\mathcal{J}^{\prime}$ of the form (4.5) in the free GAT on $X$ comprises a type judgement as above with associated element $(\varphi,(\alpha, h))$, together with a term expression $t$ such that either:

(i) $x_{j_{1}}: T_{j_{1}}, \ldots, x_{j_{k-1}}: T_{j_{k-1}} \vdash t: T_{n}$ is derivable in the free $\{s\}$-GAT on $X$; or

(ii) $t=x_{i}$ for some $i \in[n-1]$ such that $T_{n}=T_{i}$; i.e., such that $\varphi(n)=\varphi(i)$, $\alpha_{n}=\alpha_{i}$ and $h_{n}=h_{i}$.

We may thus assign to $\left[\mathcal{J}^{\prime}\right] \in \operatorname{Tm}_{T X}([\mathcal{J}])$ an element of $\operatorname{Tm}_{P S X}(\varphi,(\alpha, h))$, lying in the left- or right-hand summand according as $t$ is of the form (i) or (ii). An inductive argument like the one above now shows that this mapping is inverse to the component $\operatorname{Tm}_{P S X}(\varphi,(\alpha, h)) \rightarrow \operatorname{Tm}_{T X}\left(\kappa_{X}(\varphi,(\alpha, h))\right)$ of $\kappa_{X}$.

By transporting the monad structure of $T$ along the isomorphisms (7.1), we thus obtain a monad structure on $P S$. With respect to this structure, the maps $\eta^{P} S: S \Rightarrow P S$ and $S \eta^{P}: P \Rightarrow P S$ now become monad morphisms which in 
addition satisfy the "middle unit law" expressed by the commutativity of:

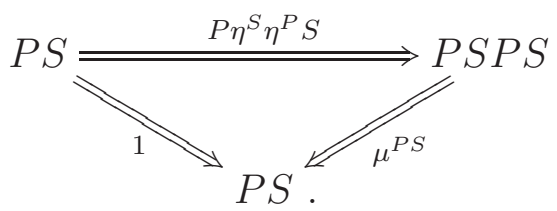

Henceforth, we shall take it that in fact $T=P S$.

7.2. Unit and multiplication. We now describe the unit and the multiplication of the monad for GATs in terms of those for the weakening-and-projection and substitution monads. The case of the unit is straightforward.

Proposition 36. For each $X \in\left[\mathcal{H}^{\text {op }}\right.$, Set $]$, the unit map $\eta_{X}^{P S}: X \rightarrow P S X$ of the monad for GATs is the composite

$$
X \stackrel{\eta_{X}^{S}}{\longrightarrow} S X \stackrel{\eta_{S X}^{P}}{\longrightarrow} P S X
$$

Proof. An immediate consequence of the fact that $\eta^{P} S$ is a monad map.

The multiplication $\mu^{P S}$ of the monad for GATs may be described in terms of those of $S$ and $P$ together with one additional datum: that of a distributive law of $S$ over $P$ in the sense of [1]. This is a natural transformation $\delta: S P \Rightarrow P S$ satisfying four axioms relating it to the units and multiplications of the monads $S$ and $P$. It may be obtained from the multiplication $\mu^{P S}$ as the composite:

$$
\delta=S P \stackrel{\eta^{P} S P \eta^{S}}{\Longrightarrow} P S P S \stackrel{\mu^{P S}}{\Longrightarrow} P S .
$$

In a moment, we shall give an explicit description of $\delta$, but first let us record how it allows us to reconstruct the multiplication of $P S$ :

Proposition 37. For each $X \in\left[\mathcal{H}^{\mathrm{op}}\right.$, Set $]$, the multiplication $\mu_{X}^{P S}: P S P S X \rightarrow$ $P S X$ of the monad for GATs is the composite

$$
P S P S X \stackrel{P \delta_{S X}}{\longrightarrow} P P S S X \stackrel{\mu^{P} \mu_{X}^{S}}{\longrightarrow} P S X .
$$

Proof. This is $(1) \Leftrightarrow(2)$ of $[1$, Proposition, Section 1].

Since we already have explicit descriptions of $\mu^{P}$ and $\mu^{S}$, this allows us to reduce the problem of giving an explicit description of $\mu^{P S}$ to that of giving one for $\delta$. Such a description is essentially an account of how the process of substituting terms into a weakened judgement may be re-expressed as the process of weakening a judgement to which substitution has already been applied. The behaviour is different depending on whether the terms we are substituting in are genuine terms or are projections onto a variable; those of the former kind induce actual substitutions, while those of the latter express the structural rule of contraction. Our description of $\delta_{X}$ will thus come in two parts, the first dealing only with actual substitutions, and the second reintroducing contraction. 
First we need an explicit description of $S P X$. Given $\alpha \in \operatorname{Inc}(n)$, a map $[\alpha] \rightarrow P X$ is determined as in the discussion following Definition 29 by its value at $\alpha(n)$ and its values at $i_{t}$ for each $i \in[\alpha(n)] \backslash \operatorname{Im} \alpha$, thus by giving:

- A pair $(\varphi \in \operatorname{Hp}(\alpha(n)), h:[\varphi] \rightarrow X) \in P X(\alpha(n))=W X(\alpha(n))$; and

- Elements $k(i) \in \operatorname{Tm}_{P X}\left(\left.\varphi\right|_{i},\left.h\right|_{i}\right)$ for each $i \in[\alpha(n)] \backslash \operatorname{Im} \alpha$,

and so we write a typical element of $\operatorname{SPX}(n)$ as $(\alpha,(\varphi, h, k))$. By Proposition 25, the set $\operatorname{Tm}_{P X}\left(\left.\varphi\right|_{i},\left.h\right|_{i}\right)$ which each $k(i)$ inhabits is the disjoint union

$$
\operatorname{Tm}_{X}(h(i))+\left\{\pi_{j}: j \in[i-1], \varphi(i)=\varphi(j), h(i)=h(j)\right\} ;
$$

we will call $(\alpha,(\varphi, h, k))$ projection-free if each $k(i)$ lies in the left-hand summand. We first describe the action of $\delta_{X}$ on projection-free elements.

Definition 38. Let $(\alpha,(\varphi, h, k)) \in S P X(n)$ be projection-free. We define the heap $\alpha^{*} \varphi \in \operatorname{Hp}(n)$ in relation form by $i \preccurlyeq_{\alpha^{*} \varphi} j$ iff $\alpha(i) \preccurlyeq_{\varphi} \alpha(j)$. Given $p \in[n]$ with $\downarrow_{\alpha^{*} \varphi}(p)=\left\{p_{1} \prec \cdots \prec p_{m}=p\right\}$, we define $\alpha_{p}^{\varphi} \in \operatorname{Inc}(m)$ by $\alpha_{p}^{\varphi}(i)=$ $\operatorname{dp}_{\varphi}\left(\alpha\left(p_{i}\right)\right)$. If now $\downarrow_{\varphi}(\alpha(p))=\left\{v_{1} \prec \cdots \prec v_{\ell}=\alpha(p)\right\}$, then it is easy to see that $i \in[\ell]$ is in the image of $\alpha_{p}^{\varphi}$ just when $v_{i}$ is in the image of $\alpha$; it follows that we have a well-defined mapping $(h+k)_{p}:\left[\alpha_{p}^{\varphi}\right] \rightarrow X$ given by

$$
(h+k)_{p}(i)=h\left(v_{i}\right) \text { and }(h+k)_{p}\left(i_{t}\right)=k\left(v_{i}\right)
$$

for $i \in[\ell]$ (on the left) and $i \in[\ell] \backslash \operatorname{Im} \alpha_{p}^{\varphi}$ (on the right). It is moreover easy to verify that $\partial\left(\alpha_{p}^{\varphi},(h+k)_{p}\right)=\left(\alpha_{q}^{\varphi},(h+k)_{q}\right)$ whenever $\alpha^{*} \varphi(p)=q$, so that we have a well-defined mapping $\left(\alpha^{\varphi}, h+k\right):\left[\alpha^{*} \varphi\right] \rightarrow S X$, and so an element $\left(\alpha^{*} \varphi,\left(\alpha^{\varphi}, h+k\right)\right) \in P S X(n)$.

Example 39. If $\alpha$ is the inc-list $0<1<5<6<7<8$ and $\varphi$ is as on the left below, then $\alpha^{*} \varphi$ and $\alpha^{\varphi}$ are as on the right.
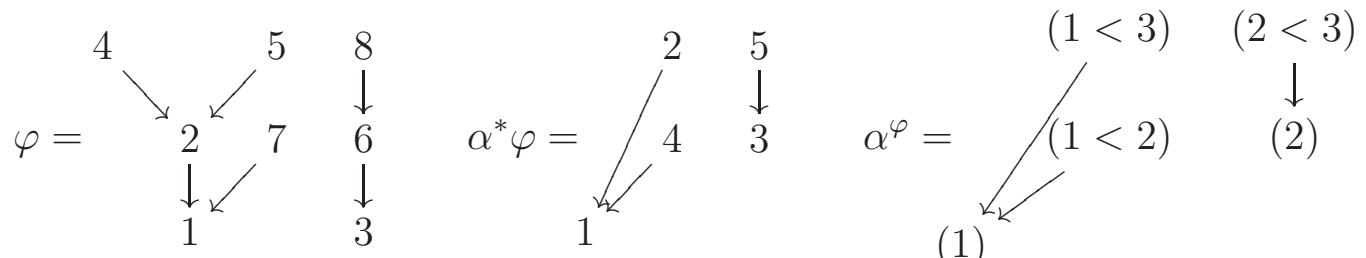

We now wish to describe the action of $\delta_{X}$ on arbitrary type-elements. As a first step, let us call $(\alpha,(\varphi, h, k)) \in S P X(n)$ nearly projection-free if the only terms $k(i)$ which are projections are ones for which $i$ is not in the image of $\varphi$ (thus leaves in the forest corresponding to $\varphi$ ). For such an element, we can still define $\left(\alpha^{*} \varphi,\left(\alpha^{\varphi}, h+k\right)\right)$ as above; the point which requires checking is that, for $p \in[n]$ with $\downarrow_{\varphi}(\alpha(p))=\left\{v_{1} \prec \cdots \prec v_{\ell}=\alpha(p)\right\}$ and $i \in[\ell] \backslash \operatorname{Im} \alpha_{p}^{\varphi}$, the element $k\left(v_{i}\right)$ should be a term of $X$ rather than a projection. But this is true since we necessarily have $i<\ell$, so that $v_{i}$ is in the image of $\varphi$ and thus a term of $X$ by assumption. With this observation in mind, we may now extend our description of the action of $\delta_{X}$ to deal with arbitrary type-elements. 
Definition 40. Given a general element $(\alpha,(\varphi, h, k)) \in S P X(n)$, let $\leq_{k}$ be the partial order generated on $[0, \alpha(n)]$ by the basic inequalities:

$$
i \leq_{k} j \text { when } j \notin \operatorname{Im} \alpha \text { and } k(j)=\pi_{i},
$$

and let $\bar{\varphi} \in \operatorname{Hp}(\alpha(n))$ be given by $\bar{\varphi}(i)=\min \left\{j: j \leq_{k} \varphi(i)\right\}$. Note that $i \leq_{k} j$ implies $\varphi(i)=\varphi(j)$ and $h(i)=h(j)$, which means that $\operatorname{dp}_{\varphi}(i)=\operatorname{dp}_{\bar{\varphi}}(i)$ for all $i \in[\alpha(n)]$ and, if $\bar{\varphi}(i)>0$, that $\partial h(i)=h(\bar{\varphi}(i))$. Thus $(\alpha,(\bar{\varphi}, h, k))$ is a welldefined element of $S P X(n)$ which is easily seen to be nearly projection-free, so that we may form $\left(\alpha^{*} \bar{\varphi},\left(\alpha^{\bar{\varphi}}, h+k\right)\right) \in P S X(n)$.

This completes our description of the action of $\delta_{X}$ on type-elements; we now prove its validity at the same time as giving the action on term-elements. For the latter, let us note that we have $\operatorname{Tm}_{S P X}(\alpha,(\varphi, h, k))=\operatorname{Tm}_{P X}(\varphi, h)$ which by Proposition 25 again is the disjoint union

$$
\operatorname{Tm}_{X}(h(\alpha(n)))+\left\{\pi_{j}: j \in[\alpha(n)-1], \varphi(\alpha(n))=\varphi(j), h(\alpha(n))=h(j)\right\} .
$$

Proposition 41. For each $X \in\left[\mathcal{H}^{\mathrm{op}}\right.$, Set $]$, the action of the distributive law $\delta_{X}$ is given on type-elements $S P X(n) \rightarrow P S X(n)$ by

$$
(\alpha,(\varphi, h, k)) \mapsto\left(\alpha^{*} \bar{\varphi},\left(\alpha^{\bar{\varphi}}, h+k\right)\right),
$$

and on term-elements $\operatorname{Tm}_{S P X}(\alpha,(\varphi, h, k)) \rightarrow \operatorname{Tm}_{P S X}\left(\alpha^{*} \bar{\varphi},\left(\alpha^{\varphi}, h+k\right)\right)$ by

$$
a \mapsto \begin{cases}a & \text { if } a \in \operatorname{Tm}_{X}(h(\alpha(n))) ; \\ \pi_{m} & \text { if } a=\pi_{j} \text { and } \min \left\{i: i \leq_{k} j\right\}=\alpha(m) ; \\ k(m) & \text { if } a=\pi_{j} \text { and } m=\min \left\{i: i \leq_{k} j\right\} \notin \operatorname{Im} \alpha .\end{cases}
$$

Proof. We prove the result for types and terms simultaneously by induction on $\alpha(n)-n$. For the base case $\alpha(n)=n$, we must have $\alpha=\iota_{n}$, and now on typeelements we have $\delta_{X}\left(\iota_{n},(\varphi, h, k)\right)=\delta_{X}\left(\eta_{P X}^{S}(\varphi, h)\right)=P \eta_{X}^{S}(\varphi, h)=(\varphi,(\iota, \tilde{h}))$, which by direct calculation from the definitions is equal to $\left(\iota_{n}^{*} \bar{\varphi},\left(\iota_{n}^{\bar{\varphi}}, h+k\right)\right)$. The argument for terms in the base case is similarly straightforward, on observing that $\leq_{k}$ in this case satisfies $i \leq_{k} j$ iff $i=j$.

Before giving the inductive step, we make an observation. Suppose given $(\varphi,(\alpha, h)) \in P S X(n)$ and $t \in \operatorname{Tm}_{P S X}\left(\left.\varphi\right|_{m},\left.(\alpha, h)\right|_{m}\right)$. We wish to describe the judgement obtained by substituting $\mathcal{J}\left(\left.\varphi\right|_{m},\left.(\alpha, h)\right|_{m}, t\right)$ into $\mathcal{J}(\varphi,(\alpha, h))$ in the free GAT on $X$. If $t=\pi_{j}$ is a projection term, then direct inspection of the bijection of Proposition 7.1 shows that the judgement obtained is $\mathcal{J}\left(\varphi^{\prime} \backslash m,(\alpha, h) \backslash\right.$ $m)$, where $\varphi^{\prime} \in \operatorname{Hp}(n)$ is defined by $\varphi^{\prime}(i)=j$ if $\varphi(i)=m$ and $\varphi^{\prime}(i)=\varphi(i)$ otherwise. On the other hand, if $t=a \in \operatorname{Tm}_{X}\left(h_{m}\left(\alpha_{m}(\# m)\right)\right)$, inspection of Proposition 7.1 shows that this substitution yields $\mathcal{J}\left(\varphi^{\prime} \backslash m,\left(\alpha^{\prime}, h^{\prime}\right) \backslash m\right)$, with $\varphi^{\prime} \in \operatorname{Hp}(n)$ and $\left(\alpha^{\prime}, h^{\prime}\right):\left[\varphi^{\prime}\right] \rightarrow S X$ given by

$$
\varphi^{\prime}(i)=\left\{\begin{array}{ll}
\varphi^{2}(i) & \text { if } \varphi(i)=m ; \\
\varphi(i) & \text { otherwise; }
\end{array} \quad\left(\alpha_{i}^{\prime}, h_{i}^{\prime}\right)= \begin{cases}\left(\alpha_{i} \epsilon_{\ell}, h_{i} \cup a\right) & \text { if } \varphi^{\# i-\ell}(i)=m \\
\left(\alpha_{i}, h_{i}\right) & \text { otherwise }\end{cases}\right.
$$


Here we write $\epsilon_{\ell}:[0, n] \rightarrow[0, n+1]$ for the unique injection whose image does not include $\ell$, and, as in the proof of Proposition 30, write $h_{i} \cup a:\left[\alpha_{i} \epsilon_{\ell}\right] \rightarrow X$ for the map which extends $h_{i}:\left[\alpha_{i}\right] \rightarrow X$ by sending $\left(\alpha_{i}(\ell)\right)_{t}$ to $a$.

We now give the inductive step of our main argument. Let $(\alpha, \ell)=(\alpha,(\varphi, h, k)) \in$ $S P X(n)$ with $\alpha(n)>n$. As in Proposition 30, we can find $\alpha(m)<j<$ $\alpha(m+1)$ and form the type-elements $\left(\alpha_{j}, \ell_{j}\right)$ and $\left(\alpha^{j}, \ell^{j}\right)$ and term-element $k(j) \in \operatorname{Tm}_{S P X}\left(\alpha^{j}, \ell^{j}\right)$. Now we have that $\ell_{j}=\left(\varphi, h, k^{\prime}\right):\left[\alpha_{j}\right] \rightarrow X$, where $k^{\prime}$ is obtained from $k$ by removing the value at $j$; and we have that $\ell^{j}=\partial^{n-m-1}\left(\ell_{j}\right)$. So by induction, applying $\delta_{X}$ to $\left(\alpha_{j}, \ell_{j}\right)$ and $\left(\alpha^{j}, \ell^{j}, k(j)\right)$ yields the elements

$$
\left(\alpha_{j}{ }^{*} \bar{\varphi},\left(\alpha_{j}^{\bar{\varphi}}, h+k^{\prime}\right)\right) \text { and }\left(\left.\alpha_{j}{ }^{*} \bar{\varphi}\right|_{m+1},\left.\left(\alpha_{j}^{\bar{\varphi}}, h+k^{\prime}\right)\right|_{m+1}, k(j)\right)
$$

of $P S X$. The judgement $\mathcal{J}(\alpha, \ell)$ of the free $\{s\}$-GAT on $P X$ is obtained by substituting $\mathcal{J}\left(\alpha^{j}, \ell^{j}, k(j)\right)$ into $\mathcal{J}\left(\alpha_{j}, \ell_{j}\right)$, whence $\delta_{X}(\alpha, \ell)$ is obtained by substituting $\mathcal{J}\left(\left.\alpha_{j}{ }^{*} \varphi\right|_{m+1},\left.\left(\alpha_{j}{ }^{\varphi}, h+k^{\prime}\right)\right|_{m+1}, k(j)\right)$ into $\mathcal{J}\left(\alpha_{j}{ }^{*} \varphi,\left(\alpha_{j}{ }^{\varphi}, h+k^{\prime}\right)\right)$ in the free GAT on $X$. Now $k(j)$ is either a projection or non-projection; applying the appropriate part of the above observation and calculating shows that, in either case, the resultant judgement is $\mathcal{J}\left(\alpha^{*} \bar{\varphi}, \alpha^{\bar{\varphi}}, h+k\right)$, so that $\delta_{X}(\alpha, \ell)=\left(\alpha^{*} \bar{\varphi}, \alpha^{\bar{\varphi}}, h+k\right)$.

Finally, we give the inductive step on term-elements. The key point is for us to extend the observation made above. Given $(\varphi,(\alpha, h)) \in P S X(n)$ and $t \in \operatorname{Tm}_{P S X}\left(\left.\varphi\right|_{m},\left.(\alpha, h)\right|_{m}\right)$ as before and also $a \in \operatorname{Tm}_{P S X}(\varphi,(\alpha, h))$, we wish to describe the result of substituting $\mathcal{J}\left(\left.\varphi\right|_{m},\left.(\alpha, h)\right|_{m}, t\right)$ into $\mathcal{J}(\varphi,(\alpha, h), a)$. If $v$ denotes the term-element representing this judgement, then direct calculation shows that

$$
v= \begin{cases}a & \text { if } a \in \operatorname{Tm}_{X}\left(h_{n}\left(\alpha_{n}(\# n)\right)\right) \\ \pi_{j} & \text { if } a=\pi_{j} \text { and } j<m ; \\ t & \text { if } a=\pi_{m} ; \\ \pi_{j-1} & \text { if } a=\pi_{j} \text { and } j>m ;\end{cases}
$$

Applying this observation together with induction and the preservation of derivations by $\delta_{X}$ now yields the inductive step on terms. The details are similar to the type-element case and so omitted.

Drawing together the results of the previous four sections, we thus obtain the main result of the paper, giving a complete characterisation of the monad for GATs on the presheaf category $\left[\mathcal{H}^{\mathrm{op}}\right.$, Cat $]$.

Theorem 42. The monad for GATs induced by the free-forgetful adjunction GAT $\leftrightarrows\left[\mathcal{H}^{\text {op }}\right.$, Set $]$ may be taken to have underlying endofunctor $P S$, where $P$ and $S$ are as in Propositions 25 and 30; unit map at $X \in\left[\mathcal{H}^{\mathrm{op}}\right.$, Set $]$ given by $\eta^{P} \eta_{X}^{S}: X \rightarrow P S X$, where $\eta^{P}$ and $\eta^{S}$ are as in Propositions 26 and 32; and multiplication map at $X \in\left[\mathcal{H}^{\mathrm{op}}\right.$, Set $]$ given by the composite

$$
P S P S X \stackrel{P \delta_{S X}}{\longrightarrow} P P S S X \stackrel{\mu^{P} \mu_{X}^{S}}{\longrightarrow} P S X,
$$

where $\mu^{P}, \mu^{S}$ and $\delta$ are as in Propositions 27, 34, and 41 respectively. 


\section{Categorical analysis}

We have now completed the main task of the paper by describing the monads for $D$-GATs for $D=\{w\},\{w, p\},\{s\}$ and $\{w, p, s\}$. The purpose of this final section is to discuss the good categorical properties that these monads have: namely, those of being local right adjoint and cartesian. These properties justify us in regarding these monads as fundamentally combinatorial in nature, and will allow us, in future work, to bring a rich body of theory [24, 18, 25, 2] to bear on the study of dependent sequent calculi. Let us begin by briefly sketching some of these applications:

- Nerve functors. Weber's "nerve theorem" [25] allows us to associate to any local right adjoint, cartesian monad on a presheaf category a nerve functor $T$-Alg $\rightarrow\left[\mathcal{E}^{\mathrm{op}}\right.$, Cat $]:$ a fully faithful embedding of the category of $T$-algebras into a presheaf category, together with a characterisation of the essential image of this functor. The importance of this is that a nerve functor can allow algebraic entities to be embedded into a geometric or topological context; hence this will allow us to explore geometric and higher-dimensional aspects of dependent sequent calculi.

- Categorical algebras. Any local right adjoint monad $T: \mathcal{C} \rightarrow \mathcal{C}$ preserves pullbacks, and so lifts to a 2-monad on the 2-category $\operatorname{Cat}(\mathcal{C})$ of categories internal to $\mathcal{C}$; an algebra for this lifted monad may be called a categorical $T$-algebra. In particular, this means that we can consider "categorical $D$ GATs". The value of this is in allowing a new approach to the coherence problem of [11], that many naturally occurring models of dependent type theory are "too weak" to be strict models of the syntax. This is resolved by observing that these models are actually categorical pseudoalgebras for the lifted 2-monad. By considering pseudomorphisms of algebras, we may perfectly well interpret the strict syntax in these weak models, thereby avoiding the use of strictifiction theorems. Among the categorical pseudoalgebras, we also find objects which represent the "syntax with substitution up to isomorphism" of [6]; and now the two-dimensional monad theory of [3] describes the relation between the strict and the weak syntax.

- Lax morphisms. As is well known, lax monoidal functors $1 \rightarrow \mathcal{V}$ from the terminal monoidal category classify monoids in $\mathcal{V}$. In a similar way, if $\mathbb{T}$ is a categorical $D$-GAT, then lax morphisms of $D$-GATs $1 \rightarrow \mathbb{T}$ correspond to models of type theory internal to $\mathbb{T}$. In particular, one may generate the free categorical D-GAT containing a D-GAT. This should be a fundamental combinatorial object, by analogy with the case of monoidal categories, where the corresponding entity is $\Delta_{+}$, the category of finite ordinals and monotone maps.

Investigating these ideas fully will be a paper in itself; for now, we merely show that the monads under investigation are indeed local right adjoint and cartesian. 
8.1. Local right adjoint and (strongly) cartesian monads. We begin by revising the notions of interest; see [4, 17, 24, 25, 2] for further discussion and applications.

Definition 43. A functor $F: \mathcal{C} \rightarrow \mathcal{D}$ is called local right adjoint if, for each $X \in \mathcal{C}$, the functor $F / X: \mathcal{C} / X \rightarrow \mathcal{D} / F X$ on slice categories is a right adjoint. A monad $T$ is called local right adjoint when its underlying endofunctor is so.

By standard pasting properties of pullbacks, if $\mathcal{C}$ has a terminal object then a functor $F: \mathcal{C} \rightarrow \mathcal{D}$ is local right adjoint just when $F / 1: \mathcal{C} / 1 \rightarrow \mathcal{D} / F 1$ is a right adjoint. Such an $F$ is thus determined by its value $F 1$ at the terminal object together with a functor $G_{1}: \mathcal{D} / F 1 \rightarrow \mathcal{C}$ left adjoint to $F / 1$. In the case $\mathcal{C}=\mathcal{D}=$ $\left[\mathcal{H}^{\mathrm{op}}\right.$, Set $]$ of interest to us, a standard categorical argument shows that giving the left adjoint $G_{1}$ is equivalent to giving an arbitrary functor $[-]: \operatorname{el}(F 1) \rightarrow$ $\left[\mathcal{H}^{\text {op }}\right.$, Set $]$. Here el $(F 1)$ is the category of elements of $F 1$, whose object set is $\Sigma_{h \in \mathcal{H}} F 1(h)$, and whose morphisms $(x \in F 1(h)) \rightarrow\left(x^{\prime} \in F 1\left(h^{\prime}\right)\right)$ are maps $f: h \rightarrow h^{\prime}$ such that $x=(F 1)(f)\left(x^{\prime}\right)$. Given $F 1$ and [-], we can reconstruct $F$ from it by the formula

$$
F X(h) \cong \sum_{x \in F 1(h)}\left[\mathcal{H}^{\mathrm{op}}, \operatorname{Set}\right]([x], X) .
$$

This expresses that an element of $F X(h)$ is an element $x$ of $F 1(h)$ together with an appropriate labelling $[x] \rightarrow X$ by elements of $X$. Thus elements of $F 1$ can be seen as encoding the "shapes" of the operations appearing in the functor $F$.

Note that the formula (8.1) expresses the functor $F(-)(h):\left[\mathcal{H}^{\text {op }}\right.$, Set $] \rightarrow$ Set as a coproduct of representables for each $h \in \mathcal{H}$. This provides an alternative characterisation of the local right adjoint endofunctors of $\left[\mathcal{H}^{\mathrm{op}}\right.$, Set $]$, and we record this result as:

Proposition 44. For an endofunctor $F$ of $\left[\mathcal{H}^{\mathrm{op}}\right.$, Set $]$, the following are equivalent:

(i) F is local right adjoint;

(ii) $F / 1:\left[\mathcal{H}^{\mathrm{op}}\right.$, Set $] \rightarrow\left[\mathcal{H}^{\mathrm{op}}\right.$, Set $] / F 1$ is a right adjoint;

(iii) There are given $F 1$ and $[-]: \operatorname{el}(F 1) \rightarrow\left[\mathcal{H}^{\mathrm{op}}\right.$, Set $]$ such that $(8.1)$ is validated;

(iv) For each $h \in \mathcal{H}$, the functor $F(-)(h):\left[\mathcal{H}^{\text {op }}\right.$, Set $] \rightarrow$ Set is a coproduct of representable functors;

(v) F preserves connected limits (i.e., all small fibre products and equalisers).

[The only part we have not discussed is the equivalence of (iv) and (v), which follows immediately from the fact that the functors $\left[\mathcal{H}^{\mathrm{op}}\right.$, Set $] \rightarrow$ Set which preserve connected limits are precisely the coproducts of representables.]

Definition 45. A natural transformation $\alpha: F \Rightarrow G: \mathcal{C} \rightarrow \mathcal{D}$ is called cartesian if all its naturality squares are pullbacks. A monad $T$ is called cartesian if it preserves pullbacks and its unit $1 \Rightarrow T$ and multiplication $T T \Rightarrow T$ are cartesian. A monad is strongly cartesian if it is cartesian and local right adjoint. 
Again, if $\mathcal{C}$ has a terminal object, then this definition simplifies: a natural transformation $\alpha$ as above is cartesian if and only if each naturality square of the following form is a pullback:

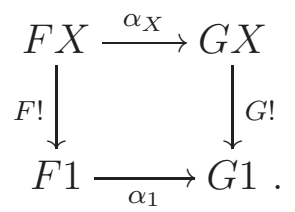

8.2. Categorical analysis. We now consider the above notions in the context of the monads $W, P, S$ and $T=P S$ for weakening, for weakening and projection, for substitution, and for GATs. We will see that $W, P$ and $S$ are all strongly cartesian, but that $T$, though local right adjoint, is not strongly cartesian.

Proposition 46. The weakening monad $W$ is strongly cartesian.

Proof. We first show $W$ is local right adjoint using the characterisation of Proposition $44(\mathrm{iv})$. For each $n \in \mathcal{H}$, we have $W(-)(n)=\sum_{\varphi \in \operatorname{Hp}(n)}\left[\mathcal{H}^{\text {op }}, \operatorname{Set}\right]([\varphi],-)$ a coproduct of representables as required. As for $W(-)\left(n_{t}\right)$, we define for each $\varphi \in \operatorname{Hp}(n)$ a presheaf $[\varphi]_{t} \in\left[\mathcal{H}^{\text {op }}\right.$, Set $]$ by taking $[\varphi]$ and adjoining a new term-element over $n \in[\varphi](\# n)$. Now $(W X)\left(n_{t}\right)=\sum_{(\varphi, h) \in P X(n)} \operatorname{Tm}_{X}(h(n)) \cong$ $\sum_{\varphi \in \operatorname{Hp}(n)}\left[\mathcal{H}^{\text {op }}, \operatorname{Set}\right]\left([\varphi]_{t}, X\right)$, whence $W(-)\left(n_{t}\right)$ is a coproduct of representables as required. We next show that $\eta^{W}: 1 \Rightarrow W$ is cartesian, thus that each naturality square (8.2) is a pullback. Evaluating at $n \in \mathcal{H}$, this says that the left square below is a pullback; while doing so at $n_{t} \in \mathcal{H}$ is the requirement that the right square be a pullback for each $A \in X(n)$.
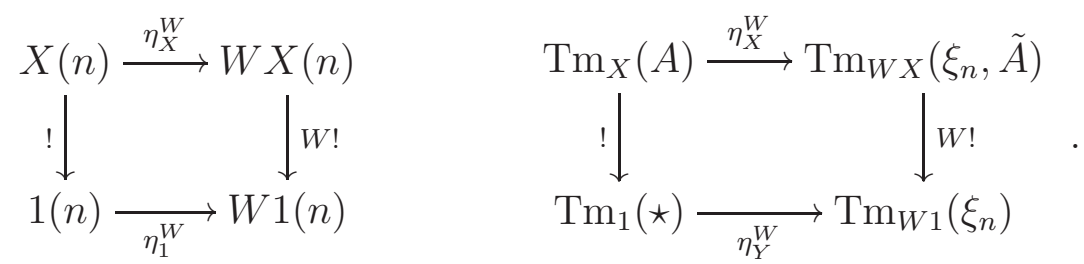

To say that the left square is a pullback is to say that each $\left(\gamma_{n}, h\right) \in W X(n)$ is of the form $\left(\gamma_{n}, \tilde{A}\right)$ for a unique $A \in X(n)$. But as we observed before Definition 20, $\left[\gamma_{n}\right]$ is the representable functor $\mathcal{H}(-, n)$, and so this follows from the Yoneda lemma. For the right-hand square, we have that $\operatorname{Tm}_{W X}\left(\gamma_{n}, \tilde{A}\right)=$ $\operatorname{Tm}_{X}(\tilde{A}(n))=\operatorname{Tm}_{X}(A)$; similarly $\operatorname{Tm}_{W 1}\left(\gamma_{n}\right)=\operatorname{Tm}_{1}(\star)$, so that both horizonal maps are isomorphisms and the square is a pullback. Finally, we show that $\mu^{W}: W W \Rightarrow W$ is cartesian, thus that the squares:
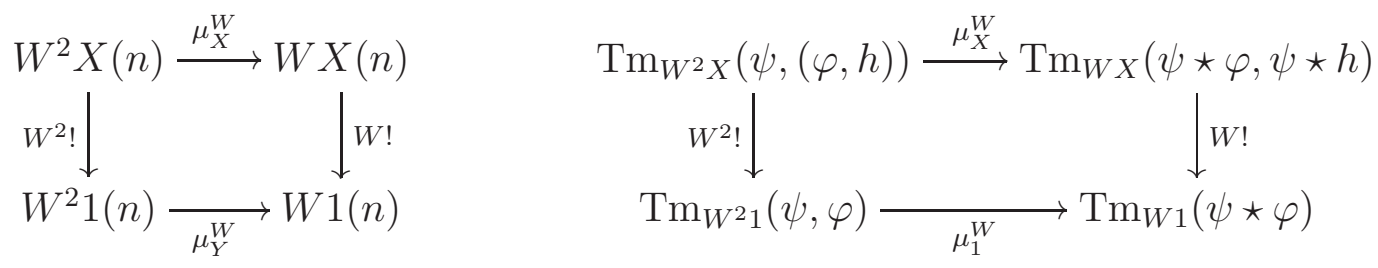
are pullbacks for all $n \in \mathcal{H}$ and all $(\psi,(\varphi, h)) \in W^{2} X(n)$. For the left square, we must show that for each $(\psi, \varphi) \in W^{2} 1(n)$ and $(\psi \star \varphi, k) \in W X(n)$, there's a unique $(\psi,(\varphi, h)) \in W^{2} X(n)$ with $k=\psi \star h$. So for each $i \in[n]$, consider the set $\downarrow_{\psi}(i)=\left\{0 \prec v_{1} \prec \cdots \prec v_{m}=i\right\}$, and now define $h_{i}:\left[\varphi_{i}\right] \rightarrow X$ by $h_{i}(j)=k\left(v_{j}\right)$. By the definition (4.6) of $\psi \star \varphi$, we see that, if $\varphi_{i}(j)=\ell$, then $(\psi \star \varphi)\left(v_{j}\right)=v_{\ell}$, whence $h_{i}(\ell)=k\left(v_{\ell}\right)=k\left((\psi \star \varphi)\left(v_{j}\right)\right)=\partial\left(k\left(v_{j}\right)\right)=\partial\left(h_{i}(j)\right)$; thus $h_{i}$ is a well-defined map. It is moreover easy to see that $\partial\left(\varphi_{i}, h_{i}\right)=\left(\varphi_{j}, h_{j}\right)$ whenever $\psi(i)=j$, so that $(\varphi, h):[\psi] \rightarrow W X$ is well-defined; finally, it it straightforward to check that $\psi \star h=k$, and that $h$ is unique with this property. Finally, for the right square, we observe that $\operatorname{Tm}_{W^{2} X}(\psi,(\varphi, h))=\operatorname{Tm}_{W X}\left(h_{n}(\# n)\right)=\operatorname{Tm}_{X}((\psi \star$ $h)(n))=\operatorname{Tm}_{W X}(\psi \star \varphi, \psi \star h)$. Thus both horizontal arrows are isomorphisms and the square is a pullback.

Proposition 47. The weakening and projection monad $P$ is strongly cartesian.

Proof. We first show that $P$ is local right adjoint. Certainly $P(-)(n)=W(-)(n)$ is a coproduct of representables; as for $P(-)\left(n_{t}\right)$, we have that

$$
P X\left(n_{t}\right)=W X\left(n_{t}\right)+\sum_{(\varphi, h) \in P X(n)}\left\{\pi_{i}: i \in[n-1], \varphi(n)=\varphi(i), h(n)=h(i)\right\},
$$

so by Proposition 46, it suffices to exhibit the right summand as a coproduct of representables. A typical element $\left(\varphi, h, \pi_{i}\right)$ of this set determines and is determined by a triple $(\partial \varphi \in \operatorname{Hp}(n-1), \partial h:[\partial \varphi] \rightarrow X, i \in[n-1])$ subject to no further conditions, so that this summand may be written as $\sum_{\varphi \in \mathrm{Hp}(n-1), i \in[n-1]}\left[\mathcal{H}^{\mathrm{op}}, \operatorname{Set}\right]([\varphi], X)$ as required. It remains to show that $\eta^{P}$ and $\mu^{P}$ are cartesian. Since $P$ agrees with $W$ on type-elements, the only extra work involves term-elements: we must show that squares of the form
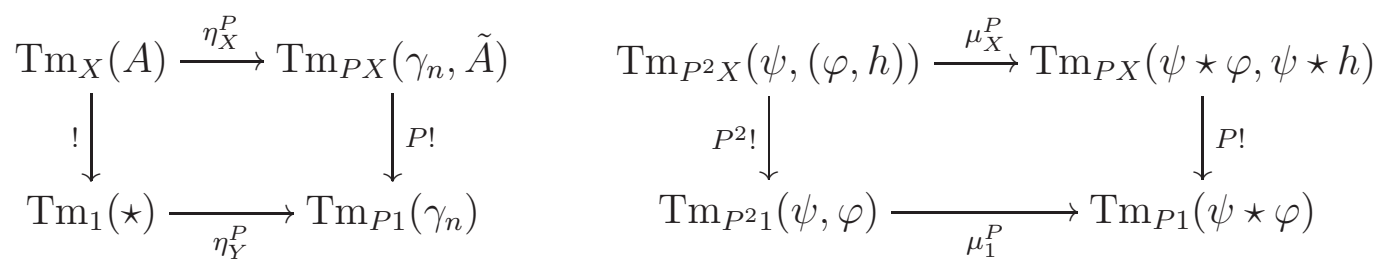

are pullbacks for all $A \in X(n)$ and for all $(\psi,(\varphi, h)) \in P^{2} X(n)$. For the left-hand square, note that $\operatorname{Tm}_{P X}\left(\gamma_{n}, \tilde{A}\right)$ contains no projection terms $\pi_{i}$, as $\gamma_{n}(n) \neq \gamma_{n}(i)$ for any $i \in[n-1]$. Thus $\operatorname{Tm}_{P X}\left(\gamma_{n}, \tilde{A}\right)=\operatorname{Tm}_{W X}\left(\gamma_{n}, \tilde{A}\right)$ and similarly for $P 1$, and so we may appeal to Proposition 46. Finally, for the right-hand square, we need only deal with the new projection terms. We must show two things:

- Given projection terms $\pi_{i}(\psi, \varphi) \in P^{2} 1(\psi, \varphi)$ and $\pi_{i} \in P X(\psi \star \varphi, \psi \star h)$, we have a valid projection term $\pi_{i}(\psi,(\varphi, h)) \in P^{2} X$; if this exists, it will clearly be the unique element sitting over $\pi_{i}(\psi, \varphi)$ and $\pi_{i}$. Since $\pi_{i}(\psi, \varphi)$ is a projection term, we already have that $\psi(n)=\psi(i)$ and $\varphi_{n}=\varphi_{i}$; and so we need only show that also $h_{n}=h_{i}$. Since $\psi(n)=\psi(i)$, we have $\partial h_{n}=\partial h_{i}$; it remains to show that $h_{i}$ and $h_{n}$ agree at $\# n=\# i$. But since 
$\pi_{i} \in P X(\psi \star \varphi, \psi \star h)$, we have $(\psi \star h)(n)=(\psi \star h)(i)$, so by definition $h_{n}(\# n)=h_{i}(\# i)$ as required.

- Given projection terms $\pi_{i}\left(\varphi_{n}\right) \in P^{2} 1(\psi, \varphi)$ and $\pi_{\psi \# n-i(n)} \in P X(\psi \star \varphi, \psi \star$ $h)$, we have a valid projection term $\pi_{i}\left(\varphi_{n}, h_{n}\right) \in P^{2} X$. Since $\pi_{i}\left(\varphi_{n}\right)$ is a projection term, we already have that $\varphi_{n}(\# n)=\varphi_{n}(i)$, so it remains to show that $h_{n}(\# n)=h_{n}(i)$. Let us write $j=\psi^{\# n-i}(n)$. Since $\pi_{j} \in P X(\psi \star \varphi, \psi \star h)$, we have $(\psi \star h)(n)=(\psi \star h)(j)$, whence $h_{n}(\# n)=(\psi \star h)(n)=(\psi \star h)(j)=$ $h_{j}(\# j)=h_{j}(i)=h_{n}(i)$, as required, where for the last step, we use the fact that $j \in \downarrow_{\psi}(n)$ and so that $h_{j}=\left.\left(h_{n}\right)\right|_{i}$.

Proposition 48. The substitution monad $S$ is strongly cartesian.

Proof. We first show that $S$ is local right adjoint. Arguing as in Proposition 46, we have that $S(-)(n)=\sum_{\alpha \in \operatorname{Inc}(n)}\left[\mathcal{H}^{\mathrm{op}}\right.$, Set $]([\varphi],-)$ is a coproduct of representables, while we may write $S(-)\left(n_{t}\right)$ as the coproduct $\sum_{\varphi \in \operatorname{Inc}(n)}\left[\mathcal{H}^{\text {op }}\right.$, Set $]\left([\alpha]_{t}, X\right)$, where the presheaf $[\alpha]_{t} \in\left[\mathcal{H}^{\text {op }}\right.$, Set $]$ is obtained by adjoining to $[\alpha]$ a new termelement over $\alpha(n) \in[\varphi](\alpha(n))$. We next show that $\eta^{S}: 1 \Rightarrow S$ is cartesian; which, as before, is to show that the squares:
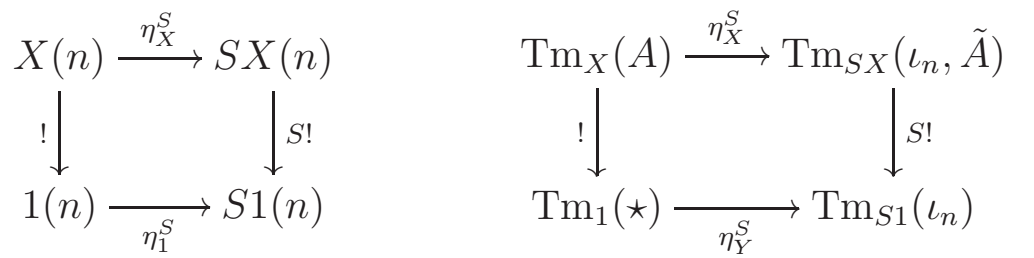

are pullbacks for each $n \in \mathcal{H}$ and each $A \in X(n)$. The argument is exactly as in Proposition 46, using the facts that $\left[\iota_{n}\right]$ is again the representable $\mathcal{H}(n,-)$, and that $\operatorname{Tm}_{S X}\left(\iota_{n}, \tilde{A}\right)=\operatorname{Tm}_{X}\left(\tilde{A}\left(\iota_{n}(n)\right)\right)=\operatorname{Tm}_{X}(A)$. Finally, we show that $\mu^{S}: S S \Rightarrow S$ is cartesian, thus that the squares:
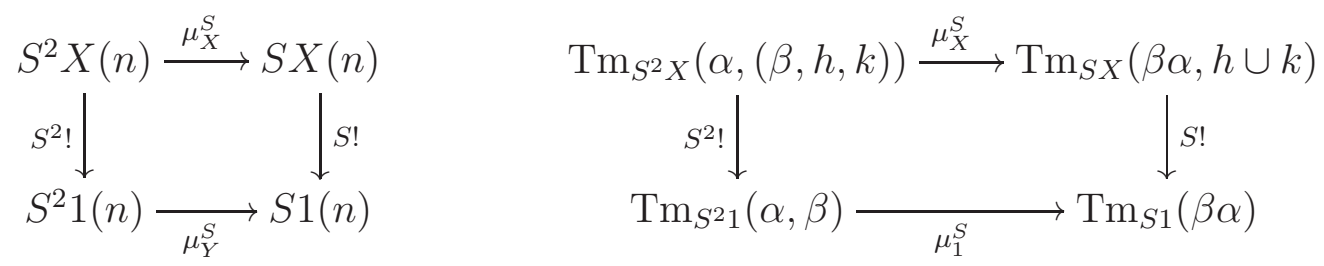

are pullbacks for all $n \in \mathcal{H}$ and all $(\alpha,(\beta, h, k)) \in S^{2} X(n)$. For the left square, we must show that for each $(\alpha, \beta) \in S^{2} 1(n)$ and $(\beta \alpha, \ell) \in S X(n)$, there's a unique $(\alpha,(\beta, h, k)) \in S^{2} X(n)$ with $\ell=h \cup k$. But this is easy: we define $h(i)=\ell(i)$ for $i \in[\beta(\alpha(n))]$ and $h\left(i_{t}\right)=\ell\left(i_{t}\right)$ for $i \in[\beta(\alpha(n))] \backslash \operatorname{Im} \beta$, and define $k(j)=\ell\left(\beta(j)_{t}\right)$ for $j \in[\alpha(n)] \backslash \operatorname{Im} \alpha$. It is easy to see that this is well-defined, that $h \cup k=\ell$, and that $h$ and $k$ are unique with this property. Finally, for the right square, we observe that $\operatorname{Tm}_{S^{2} X}(\alpha,(\beta, h, k))=\operatorname{Tm}_{S X}(\beta, h)=\operatorname{Tm}_{X}(\beta(\alpha(n)))=$ $\operatorname{Tm}_{S X}(\beta \alpha, h \cup k)$. Thus both horizontal arrows are isomorphisms and the square is a pullback. 
We conclude by considering the categorical properties of the monad $T=P S$ for GATs. One might expect that $T$, like its constituent monads $P$ and $S$, would be strongly cartesian. However, this turns out not to be the case.

Proposition 49. The monad $T$ for GATs is local right adjoint, but not strongly cartesian.

Proof. The underlying endofunctor $T=P S$ is the composite of two local right adjoint functors, and so itself is local right adjoint. Similarly, the unit $\eta^{P S}=$ $\eta^{P} S \circ \eta^{S}: 1 \Rightarrow P S$ is the composite of two cartesian natural transformations and so cartesian. However, the same is not true of the multiplication. The problem is that the distributive law $\delta: S P \Rightarrow P S$ is not a cartesian natural transformation; it follows that $\mu^{P S}$ is not either. Indeed, if it were, then both components of (7.4) would be cartesian (the first since $\eta^{P}$ and $\eta^{S}$ are and $P S$ preserves pullbacks), and hence the composite $\delta$ would be, too. It remains to prove that $\delta$ is not cartesian. Let $X \in\left[\mathcal{H}^{\text {op }}\right.$, Set $]$ be generated by a single element $A \in X(1)$, and consider the square on the left in:
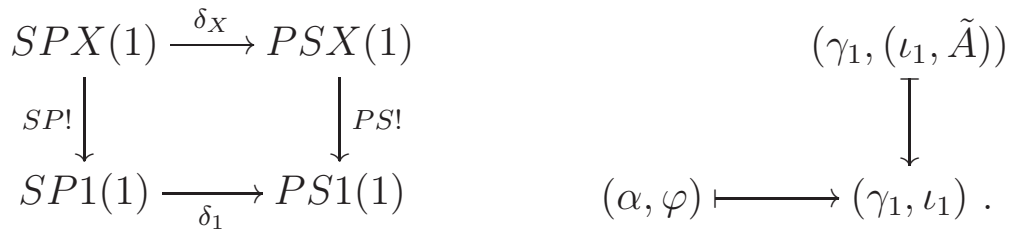

We will show that this square is not a pullback. Let $\alpha \in \operatorname{Inc}(1)$ be given by $\alpha(0)=0$ and $\alpha(1)=2$, and let $\varphi \in \operatorname{Hp}(\alpha(1))=\operatorname{Hp}(2)$ be given by $\varphi(2)=$ $\varphi(1)=\varphi(0)=0$. These data give rise to a projection-free term $(\alpha, \varphi)$ in $S P 1(1)$, and easily $\alpha^{*} \varphi=\gamma_{1}$ and $\alpha^{\varphi}=\iota_{1}$. Thus we have a diagram of elements as on the right above; but there can be no element of $S P X(1)$ forming a cone over it. For indeed, such an element would have to be a projection-free element $(\alpha,(\varphi, h, k))$, where $h:[\varphi] \rightarrow X$ and $k$ comprises an element $k(1) \in \operatorname{Tm}_{X}(h(1))$; but since $X$ has no term-elements, this is impossible.

Remark 50. It is probably worth explaining what the above failure of cartesianness means in proof-theoretic terms. Consider the following pattern of derivation. Take a type judgement $\vdash A$ type. Weaken it with respect to a closed type $B$ to obtain $x: B \vdash A$ type. Now substitute in a closed term $\vdash t: B$. The result is, of course, once again just $\vdash A$ type. The basic data for this derivation - the types $A$ and $B$ and the term $t$-correspond to an element $(\alpha,(\varphi, h, k)) \in S P X(1)$, where $\alpha$ and $\varphi$ are as in the preceding proof. The result of the derivation - the judgement $\vdash A$ type is the resultant element $\delta_{X}(\alpha,(\varphi, h, k))=\left(\gamma_{1},\left(\iota_{1}, \tilde{A}\right)\right) \in$ $P S X(1)$. When $X=1$, this reduces to $\delta_{1}(\alpha, \varphi)=\left(\gamma_{1}, \iota_{1}\right)$ and in fact $(\alpha, \varphi)$ is the unique preimage of $\left(\gamma_{1}, \iota_{1}\right)$ under $\delta_{1}$. Thus if $\delta$ were to be cartesian, then each $\left(\gamma_{1},\left(\iota_{1}, \tilde{A}\right)\right)$ would also have to have a unique preimage of the form $(\alpha,(\varphi,-,-))$. But this would be to say that there were a unique derivation of $\vdash A$ type given by weakening and then substituting as above. This is clearly is not so: there is one such derivation for each closed type $B$ and each term $t$ of that type. 
Thus, finally, the reason for the failure of cartesianness is that, in the presence of weakening, application of substitution may destroy information like that of $B$ and $t$ in the above example. Thus the failure is a failure of linearity. What is perhaps remarkable is that substitution is linear in this sense with respect to the theory without weakening; it is only the interaction of substitution with weakening that causes the problem.

\section{REFERENCES}

[1] Beck, J. Distributive laws. In Seminar on Triples and Categorical Homology Theory (Zürich, 1966/67), vol. 80 of Lecture Notes in Mathematics. Springer, 1969, pp. 119-140.

[2] Berger, C., Melliès, P.-A., And Weber, M. Monads with arities and their associated theories. Journal of Pure and Applied Algebra 216 (2012), 2029-2048.

[3] Blackwell, R., Kelly, G. M., And Power, A. J. Two-dimensional monad theory. Journal of Pure and Applied Algebra 59, 1 (1989), 1-41.

[4] Carboni, A., and Johnstone, P. Connected limits, familial representability and Artin glueing. Mathematical Structures in Computer Science 5, 4 (1995), 441-459.

[5] Cartmell, J. Generalised algebraic theories and contextual categories. Annals of Pure and Applied Logic 32 (1986), 209-243.

[6] Curien, P.-L. Substitution up to isomorphism. Fundamenta Informaticae 19, 1-2 (1993), $51-85$.

[7] DyBJer, P. Internal type theory. In Types for proofs and programs (Torino, 1995), vol. 1158 of Lecture Notes in Computer Science. Springer, 1996, pp. 120-134.

[8] Ehrhard, T. Une sémantique catégorique des type dépendents. Application au Calcul des Constructions. PhD thesis, Université Paris VII, 1988.

[9] Harmer, R., Hyland, M., And Melliès, P.-A. Categorical combinatorics for innocent strategies. In LICS (2007), IEEE Computer Society, pp. 379-388.

[10] Hofmann, M. Extensional concepts in intensional type theory. PhD thesis, University of Edinburgh, 1995.

[11] Hofmann, M. On the interpretation of type theory in locally cartesian closed categories. In Computer science logic (Kazimierz, 1994), vol. 933 of Lecture Notes in Computer Science. Springer, 1995, pp. 427-441.

[12] Hyland, J. M., AND OnG, L. On full abstraction for PCF: I, II and III. Information and Computation 163, 2 (2000), 285-408.

[13] Hyland, J. M., AND Pitts, A. The theory of constructions: categorical semantics and topos-theoretic models. In Categories in computer science and logic (Boulder, 1987), vol. 92 of Contemporary Mathematics. American Mathematical Society, 1989, pp. 137-199.

[14] JacoBs, B. Comprehension categories and the semantics of type dependency. Theoretical Computer Science 10\%, 2 (1993), 169-207.

[15] Joyal, A. Foncteurs analytiques et espèces de structures. In Combinatoire énumérative (Montreal, 1985), vol. 1234 of Lecture Notes in Mathematics. Springer, 1986, pp. 126-159.

[16] Kock, J., Joyal, A., Batanin, M., and Mascari, J.-F. Polynomial functors and opetopes. Advances in Mathematics 224, 6 (2010), 2690-2737.

[17] Leinster, T. Higher operads, higher categories, vol. 298 of London Mathematical Society Lecture Note Series. Cambridge University Press, 2004.

[18] Leinster, T. Operads in higher-dimensional category theory. Theory and Applications of Categories 12, 3 (2004), 73-194.

[19] Mac Lane, S. Categories for the working mathematician, vol. 5 of Graduate Texts in Mathematics. Springer, 1971. 
[20] Pitts, A. M. Categorical logic. In Handbook of Logic in Computer Science, Volume 5. Algebraic and Logical Structures, S. Abramsky, D. M. Gabbay, and T. S. E. Maibaum, Eds. Oxford University Press, 2000, pp. 39-128.

[21] Street, R. The petit topos of globular sets. Journal of Pure and Applied Algebra 154, 1-3 (2000), 299-315.

[22] Streicher, T. Investigations into intensional type theory. Habilitationsschrift, LudwigMaximilians-Universität München, 1993.

[23] Univalent Foundations Program. Homotopy Type Theory: Univalent Foundations of Mathematics. http://homotopytypetheory.org/book, Institute for Advanced Study, 2013.

[24] Weber, M. Generic morphisms, parametric representations and weakly Cartesian monads. Theory and Applications of Categories 13 (2004), 191-234.

[25] WeBer, M. Familial 2-functors and parametric right adjoints. Theory and Applications of Categories 18 (2007), 665-732.

[26] Weber, M. Multitensors as monads on categories of enriched graphs. Theory and Applications of Categories 28 (2013), No. 26, 857-932.

Department of Mathematics, Macquarie University, NSW 2109, Australia

E-mail address: richard.garner@mq.edu.au 